\title{
P Street Corridor, Phase 1 Methods
}

Research Fellow:

Catherine De Almeida

Assistant Professor of Landscape Architecture

University of Nebraska-Lincoln

Research Assistants:

Hannah LoPresto and Brandon Zambrano

BLA Candidates

University of Nebraska-Lincoln

Firm Liaisons:

Allyson Mendenhall

Principal

Design Workshop

Karen Nalow

Principal

The Clark Enersen Partners

This investigation was conducted as part of the Landscape Architecture Foundation's 2018 Case Study Investigation (CSI) program. CSI matches faculty-student research teams with design practitioners to document the benefits of exemplary high-performing landscape projects. Teams develop methods to quantify environmental, social, and economic benefits and produce Case Study Briefs for LAF's Landscape Performance Series.

The full case study can be found at:

https://landscapeperformance.org/case-study-briefs/p-street-corridor

To cite:

De Almeida, Catherine, Hannah LoPresto, and Brandon Zambrano. "P Street Corridor, Phase 1 Methods." Landscape Performance Series. Landscape Architecture Foundation, 2018.

https://doi.org/10.31353/cs1341 


\section{Overview}

The P Street Corridor project is a revitalized 6-block urban downtown streetscape in Lincoln, Nebraska that has the primary goal of transforming unpleasant, underused spaces to grant greater public access. Located just blocks away from the University of Nebraska-Lincoln City campus, the corridor is within a half-mile of various museums, restaurants, and Nebraska's State Capitol building (see Figure: Site Location and Context). The corridor was redesigned to enhance the pedestrian streetscape experience and establish environmental integrity through increased tree canopy coverage, stormwater management, and native planting strategies. The project focuses on runoff capture and reduced irrigation cost for roadside planted bioswales. The P Street redesign was done by Design Workshop, and construction administration was executed by a local Lincoln firm, Clark Enersen Partners. The design firm's baseline data collection was extensive-covering themes such as user perception, impervious surfaces, and property values, which have informed many of the benefits within this case study. While developing the master plan, the design firm organized a community engagement process that provided excellent baseline data for social benefits. Our survey replicates the design firm's preproject survey (with a few additions), which collected post occupancy social data in order to make direct comparisons of user perception before and after the corridor redesign.

The following Methods document showcases a broad range of data collection and analysis methodologies. Beyond user surveys, the research team has taken on-site inventory of tree $\mathrm{DBH}$, pedestrian crossing times, building vacancy, and locally owned businesses. These collection strategies allow for clear analytical comparison to pre-project conditions through the use of the design firm's baseline data and recorded city data.

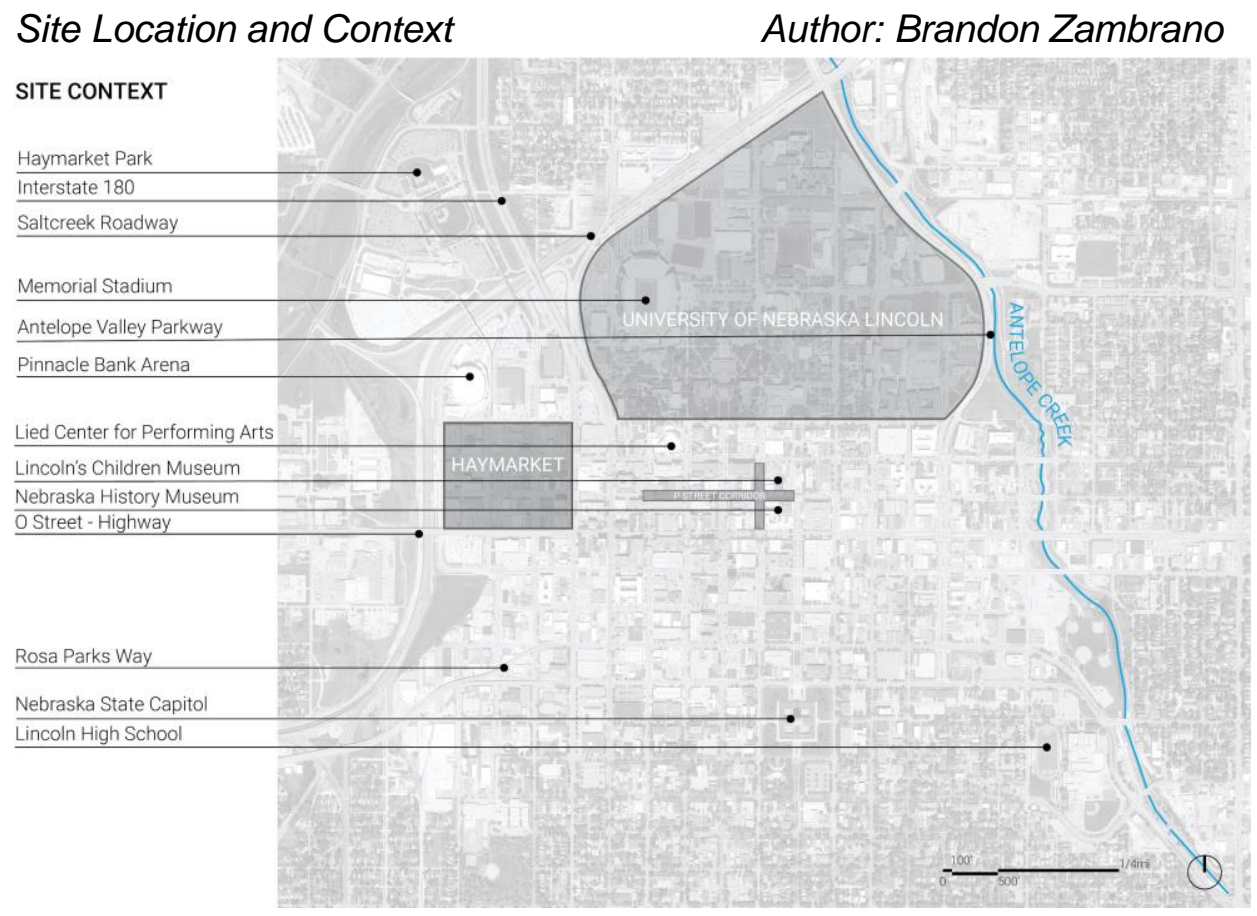


Table of Contents

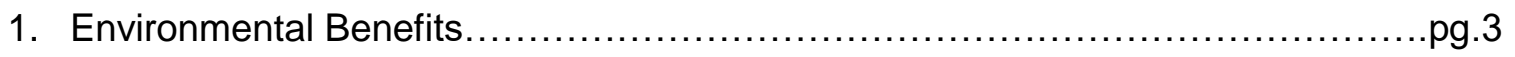

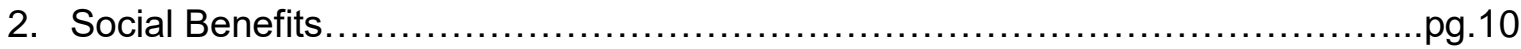

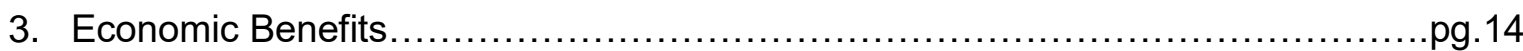

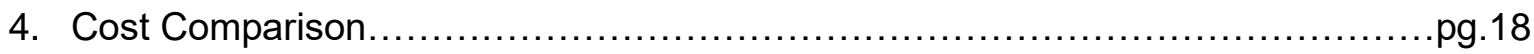

5. Appendix A: P Street Survey and Survey Results.................................pg.21

6. Appendix B: Locally Owned Streetfront, No Benefit Found...........................pg.32

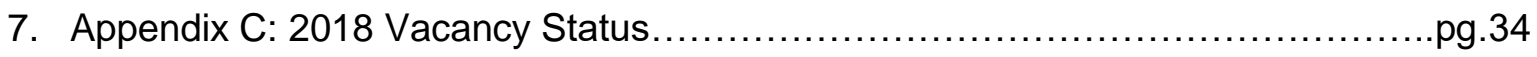

\section{Environmental Benefits}

- Sequesters $23,378 \mathrm{lbs}$ of atmospheric carbon, equivalent to driving 26,000 miles in a single-passenger vehicle, and intercepts approximately 36,600 gallons of stormwater runoff annually in existing and newly-planted trees.

Figure 1.1: Tree Inventory Author: Brandon Zambrano

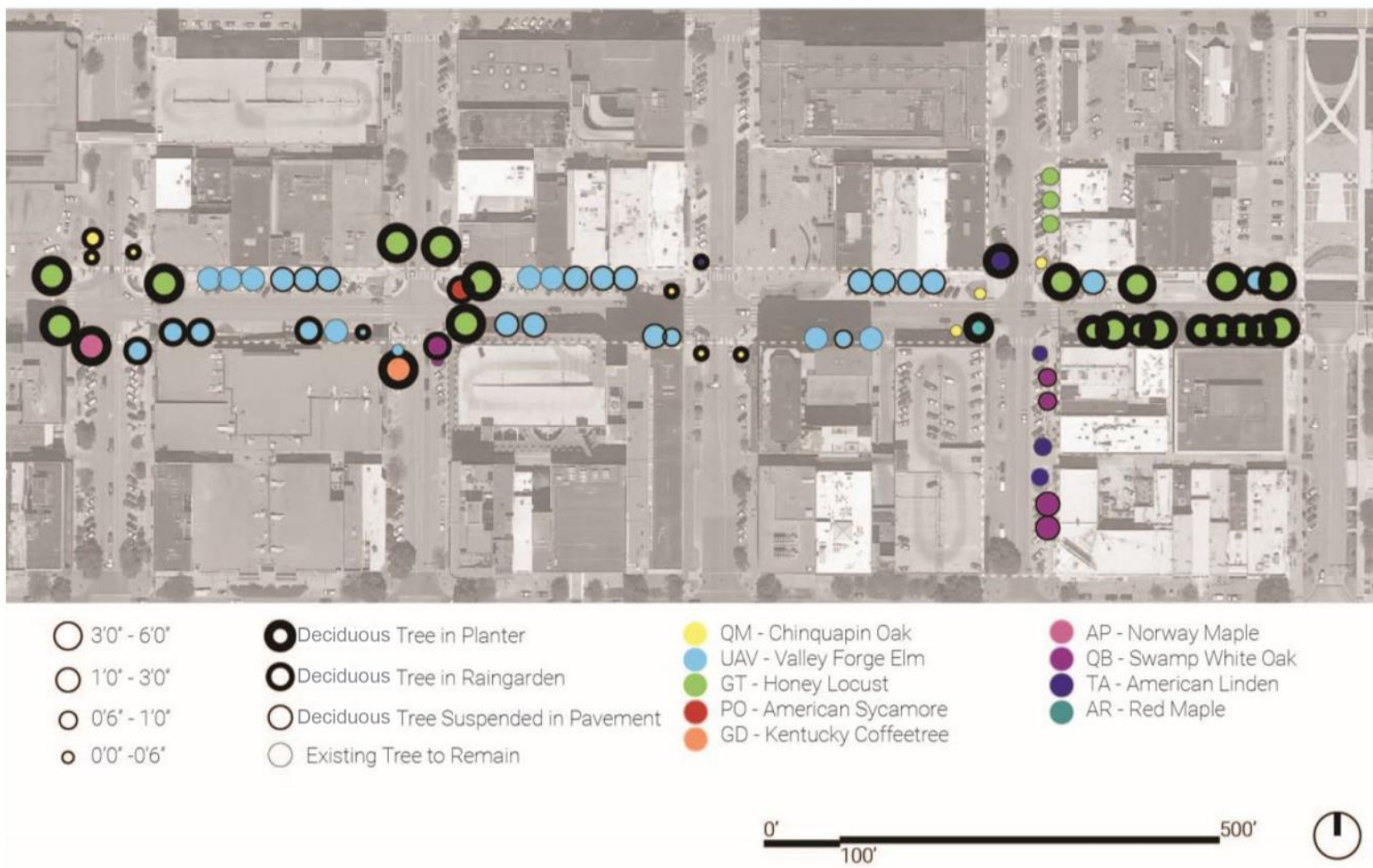

Methods:

Although some existing on-site trees were preserved, many new street trees were planted either in planters or Silva Cells. All tree species on site, both newly planted and original, had their 
circumference at breast height ( $4.5 \mathrm{ft}$. up from the tree base) and location inventoried and verified (see Figure 1.1 above). Circumference was then used to determine the average diameter of each species (see Table 1.1). i-Tree Streets was used to estimate the amount of carbon sequestered and intercepted gallons of stormwater captured per year by these trees. When a specific species was not available, the genus was used instead. The tree species, average $\mathrm{DBH}$, and land use was entered into this calculator (see Table 1.2 and 1.5).

For carbon sequestration, the amount of atmospheric carbon sequestered per year was then multiplied by the number of specific species of trees found on site. All totals were added up to obtain a total annual reduction of atmospheric carbon on-site. A calculation of just the existing tree count numbers was charted (see Table 1.3) to subtract from the on-site total to find the approximate reduction of atmospheric carbon contributed by only newly-planted trees (see Table 1.4).

The stormwater interception calculation encompasses stormwater intercepted by tree canopies. After tree species, average $\mathrm{DBH}$, and land use was entered into the calculator (see Table 1.5), the amount of intercepted gallons per year was then multiplied by the number of specific tree species found on site. All totals were added to obtain a total annual stormwater interception value. A calculation of the existing tree count numbers was charted (see Table 1.6) to subtract from the on-site total to find the approximate gallons of stormwater intercepted by only newlyplanted trees (see Table 1.7).

\section{Calculations:}

Table 1.1: Street Tree Circumference and Average Diameter (DBH) - All

\begin{tabular}{|c|c|c|c|c|c|c|c|c|c|c|}
\hline & $\begin{array}{l}\text { Chinquapin } \\
\text { Oak } \\
\text { Quercus } \\
\text { muehlenbergii }\end{array}$ & $\begin{array}{l}\text { Valley } \\
\text { Forge Elm } \\
\text { Ulmus } \\
\text { Americana } \\
\text { 'Valley } \\
\text { Forge' }\end{array}$ & $\begin{array}{l}\text { Honey } \\
\text { Locust } \\
\text { Gleditsia } \\
\text { triacanthos }\end{array}$ & $\begin{array}{l}\text { American } \\
\text { Sycamore } \\
\text { Platanus } \\
\text { occidentalis }\end{array}$ & $\begin{array}{l}\text { Norway } \\
\text { Maple } \\
\text { Acer } \\
\text { platanoides }\end{array}$ & $\begin{array}{l}\text { Kentucky } \\
\text { coffeetree } \\
\text { Gymnocladus } \\
\text { dioicus }\end{array}$ & $\begin{array}{l}\text { Swamp } \\
\text { White Oak } \\
\text { Quercus } \\
\text { bicolor }\end{array}$ & $\begin{array}{l}\text { American } \\
\text { Linden } \\
\text { Tilia } \\
\text { americana }\end{array}$ & $\begin{array}{l}\text { Red } \\
\text { Maple } \\
\text { Acer } \\
\text { rubrum }\end{array}$ & $\begin{array}{l}\text { Live oak } \\
\text { Quercus } \\
\text { virginiana }\end{array}$ \\
\hline $\begin{array}{l}\text { Specimen } 1 \\
\text { Circumference }\end{array}$ & $5^{\prime \prime}$ & $1^{\prime}-4^{\prime \prime}$ & $3^{\prime}-3^{\prime \prime}$ & $10^{\prime \prime}$ & $3^{\prime}-10^{\prime \prime}$ & 4'-1" & 3" & $4^{\prime}-6^{\prime \prime}$ & 2'-11" & $1^{\prime}$ \\
\hline $\begin{array}{l}\text { Specimen } 2 \\
\text { Circumference }\end{array}$ & 4.5" & $1^{\prime}-3^{\prime \prime}$ & $3^{\prime}-7^{\prime \prime}$ & $1^{\prime}-.5 "$ & & & 3" & & & \\
\hline $\begin{array}{l}\text { Specimen } 3 \\
\text { Circumference }\end{array}$ & $3.5^{\prime \prime}$ & 2'-7"' & $5^{\prime}-7^{\prime \prime}$ & & & & $2.5^{\prime \prime}$ & & & \\
\hline $\begin{array}{l}\text { Specimen } 4 \\
\text { Circumference }\end{array}$ & $5^{\prime \prime}$ & $1^{\prime}-4^{\prime \prime}$ & $4^{\prime}-4^{\prime \prime}$ & & & & 2" & & & \\
\hline $\begin{array}{l}\text { Specimen } 5 \\
\text { Circumference }\end{array}$ & 4" & 1'-9.5" & $4^{\prime}-2^{\prime \prime}$ & & & & & & & \\
\hline $\begin{array}{l}\text { Specimen } 6 \\
\text { Circumference }\end{array}$ & 3" & $1^{\prime}-4^{\prime \prime}$ & $3^{\prime}-9.5^{\prime \prime}$ & & & & & & & \\
\hline $\begin{array}{l}\text { Specimen } 7 \\
\text { Circumference }\end{array}$ & 3" & $1^{\prime}-6 "$ & $4^{\prime}-5^{\prime \prime}$ & & & & & & & \\
\hline $\begin{array}{l}\text { Specimen } 8 \\
\text { Circumference }\end{array}$ & $5^{\prime \prime}$ & $1^{\prime}-3^{\prime \prime}$ & $8.5^{\prime \prime}$ & & & & & & & \\
\hline
\end{tabular}




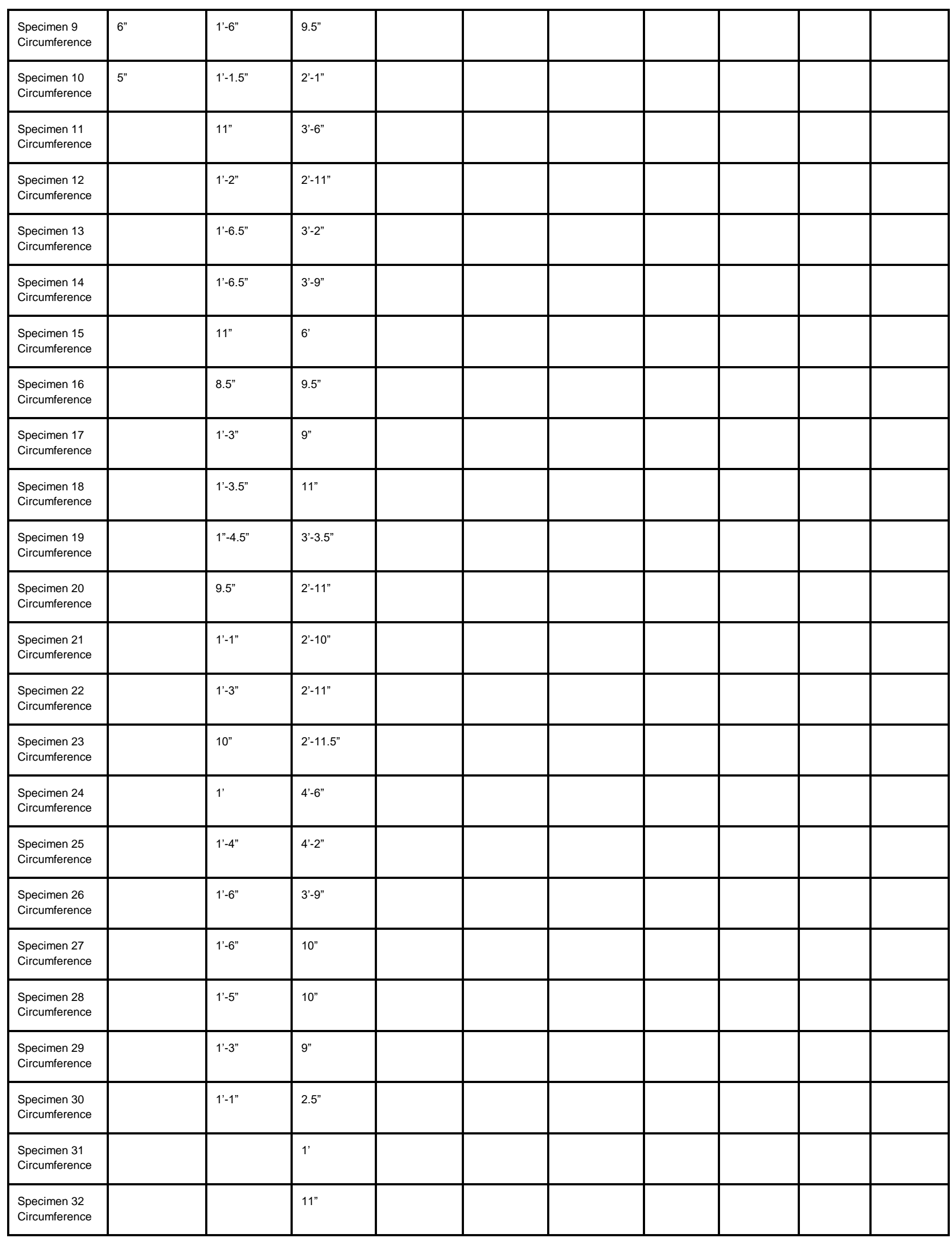




\begin{tabular}{|c|c|c|c|c|c|c|c|c|c|c|}
\hline $\begin{array}{l}\text { Average } \\
\text { Circumference }\end{array}$ & 4.4" & 15.5" & $32.39 "$ & 11.25" & $46 "$ & $49 "$ & 2.63" & $54 "$ & $35 "$ & 12" \\
\hline $\begin{array}{l}\text { Calculation } \\
\mathrm{C}=\pi \mathrm{rd}\end{array}$ & $4.4^{\prime \prime}=\pi d$ & $15.5^{\prime \prime}=\pi d$ & $32.39 "=\pi d$ & $11.25^{\prime \prime}=\pi d$ & $46 "=\pi d$ & $49 "=\pi d$ & $2.63^{\prime \prime}=\pi d$ & $54 "=\pi d$ & $35 "=\pi d$ & $12 "=\pi d$ \\
\hline Average DBH & $1.40 "$ & $4.93 "$ & 10.31" & $3.58 "$ & 14.64" & $15.60 "$ & $0.84 "$ & 17.19" & 11.14" & 3.82" \\
\hline
\end{tabular}

Table 1.2: Total Street Tree Atmospheric Carbon Reduction Per Year - All

\begin{tabular}{|c|c|c|c|c|c|c|}
\hline Species & Average DBH (inches) & Land Use & $\begin{array}{l}\text { Atmospheric } \\
\text { Carbon } \\
\text { Reduction Per } \\
\text { Year (lbs) }\end{array}$ & \# of Trees & Calculation & $\begin{array}{l}\text { Total Atmospheri } \\
\text { Carbon Reduction } \\
\text { by Species Per } \\
\text { Year (Ibs) }\end{array}$ \\
\hline $\begin{array}{l}\text { Chinquapin Oak } \\
\text { Quercus muehlenbergii }\end{array}$ & 1.40 & $\begin{array}{l}\text { Industrial or Large } \\
\text { Commercial Business }\end{array}$ & 7 & 10 & $7 \times 10=$ & 70 \\
\hline $\begin{array}{l}\text { Valley Forge Elm } \\
\text { Ulmus Americana 'Valley } \\
\text { Forge' }\end{array}$ & 4.93 & $\begin{array}{l}\text { Industrial or Large } \\
\text { Commercial Business }\end{array}$ & 92 & 30 & $92 \times 30=$ & 2,760 \\
\hline $\begin{array}{l}\text { Honey Locust } \\
\text { Gleditsia triacanthos }\end{array}$ & 10.31 & $\begin{array}{l}\text { Industrial or Large } \\
\text { Commercial Business }\end{array}$ & 535 & 32 & $535 \times 32=$ & 17,120 \\
\hline $\begin{array}{l}\text { American Sycamore } \\
\text { Platanus occidentalis }\end{array}$ & 3.58 & $\begin{array}{l}\text { Industrial or Large } \\
\text { Commercial Business }\end{array}$ & 87 & 2 & $87 \times 2=$ & 174 \\
\hline $\begin{array}{l}\text { Norway Maple } \\
\text { Acer platanoides }\end{array}$ & 14.64 & $\begin{array}{l}\text { Industrial or Large } \\
\text { Commercial Business }\end{array}$ & 754 & 1 & $754 \times 1=$ & 754 \\
\hline $\begin{array}{l}\text { Kentucky coffeetree } \\
\text { Gymnocladus dioicus }\end{array}$ & 15.6 & $\begin{array}{l}\text { Industrial or Large } \\
\text { Commercial Business }\end{array}$ & 863 & 1 & $863 \times 1=$ & 863 \\
\hline $\begin{array}{l}\text { Swamp White Oak } \\
\text { Quercus bicolor }\end{array}$ & 0.84 & $\begin{array}{l}\text { Industrial or Large } \\
\text { Commercial Business }\end{array}$ & 7 & 4 & $7 \times 4=$ & 28 \\
\hline $\begin{array}{l}\text { American Linden } \\
\text { Tilia americana }\end{array}$ & 17.19 & $\begin{array}{l}\text { Industrial or Large } \\
\text { Commercial Business }\end{array}$ & 966 & 1 & $966 \times 1=$ & 966 \\
\hline $\begin{array}{l}\text { Red Maple } \\
\text { Acer rubrum }\end{array}$ & 11.14 & $\begin{array}{l}\text { Industrial or Large } \\
\text { Commercial Business }\end{array}$ & 547 & 1 & $547 \times 1=$ & 547 \\
\hline \multirow{2}{*}{$\begin{array}{l}\text { Live oak } \\
\text { Quercus } \\
\text { virginiana }\end{array}$} & 3.82 & $\begin{array}{l}\text { Industrial or Large } \\
\text { Commercial Business }\end{array}$ & 96 & 1 & $96 \times 1=$ & 96 \\
\hline & & & & & & $\begin{array}{l}\text { Total Atmospheric } \\
\text { Carbon Reduction } \\
\text { On Site Per Year } \\
\text { (lbs): } 23,378\end{array}$ \\
\hline
\end{tabular}

Table 1.3:E Atmospheric Carbon Reduction - Existing Street Trees

\begin{tabular}{|l|l|l|l|l|l|l|}
\hline Species & Average DBH (inches) & Land Use & $\begin{array}{l}\text { Atmospheric } \\
\text { Carbon } \\
\text { Reduction Per } \\
\text { Year (lbs) }\end{array}$ & \# of Trees & $\begin{array}{l}\text { Calculation } \\
\begin{array}{l}\text { Total Atmospheric } \\
\text { Carbon Reduction } \\
\text { by Species Per } \\
\text { Year (Ibs) }\end{array}\end{array}$ \\
\hline $\begin{array}{l}\text { Chinquapin Oak } \\
\text { Quercus muehlenbergii }\end{array}$ & 1.40 & $\begin{array}{l}\text { Industrial or Large } \\
\text { Commercial Business }\end{array}$ & 7 & 1 & $7 \times 1=$ & 7 \\
\hline $\begin{array}{l}\text { Honey Locust } \\
\text { Gleditsia triacanthos }\end{array}$ & 10.31 & $\begin{array}{l}\text { Industrial or Large } \\
\text { Commercial Business }\end{array}$ & 535 & 19 & $535 \times 19=$ & 10,165 \\
\hline $\begin{array}{l}\text { Norway Maple } \\
\text { Acer platanoides }\end{array}$ & 14.64 & $\begin{array}{l}\text { Industrial or Large } \\
\text { Commercial Business }\end{array}$ & 754 & 1 & $754 \times 1=$ & 754 \\
\hline
\end{tabular}




\begin{tabular}{|l|l|l|l|l|l|l|}
\hline $\begin{array}{l}\text { American Linden } \\
\text { Tilia americana }\end{array}$ & 17.19 & $\begin{array}{l}\text { Industrial or Large } \\
\text { Commercial Business }\end{array}$ & 966 & 1 & $966 \times 1=$ & 966 \\
\hline $\begin{array}{l}\text { Red Maple } \\
\text { Acer rubrum }\end{array}$ & 11.14 & $\begin{array}{l}\text { Industrial or Large } \\
\text { Commercial Business }\end{array}$ & 547 & 1 & $547 \times 1=$ & 547 \\
\hline & & & & & & $\begin{array}{l}\text { Existing Tree } \\
\text { Total Atmospheric } \\
\text { Carbon Reduction } \\
\text { Per Year (lbs): } \\
12,439\end{array}$ \\
\hline
\end{tabular}

Table 1.4: Atmospheric Carbon Reduction - New Street Trees

\begin{tabular}{|l|l|l|l|}
\hline $\begin{array}{l}\text { Total Atmospheric Carbon Reduction } \\
\text { On Site Per Year (lbs): }\end{array}$ & $\begin{array}{l}\text { Existing Tree Atmospheric Carbon } \\
\text { Reduction On Site Per Year (lbs) }\end{array}$ & Calculation & $\begin{array}{l}\text { New Tree Atmospheric Carbon } \\
\text { Reduction On Site Per Year (Ibs) }\end{array}$ \\
\hline 23,378 & 12,439 & $23,378-12,439=$ & $\mathbf{1 0 , 9 3 9}$ \\
\hline
\end{tabular}

Table 1.5: Street Tree Stormwater Runoff Interception Per Year - Total

\begin{tabular}{|c|c|c|c|c|c|c|}
\hline Species & Average DBH (inches) & Land Use & $\begin{array}{l}\text { Stormwater } \\
\text { Runoff } \\
\text { Interception } \\
\text { Per Year (gal) }\end{array}$ & \# of Trees & Calculation & $\begin{array}{l}\text { Total Stormwater } \\
\text { Runoff Interception } \\
\text { by Species Per } \\
\text { Year (gal) }\end{array}$ \\
\hline $\begin{array}{l}\text { Chinquapin Oak } \\
\text { Quercus muehlenbergii }\end{array}$ & 1.40 & $\begin{array}{l}\text { Industrial or Large } \\
\text { Commercial Business }\end{array}$ & 17 & 10 & $17 \times 10=$ & 170 \\
\hline $\begin{array}{l}\text { Valley Forge Elm } \\
\text { Ulmus Americana 'Valley } \\
\text { Forge' }\end{array}$ & 4.93 & $\begin{array}{l}\text { Industrial or Large } \\
\text { Commercial Business }\end{array}$ & 130 & 30 & $130 \times 30=$ & 3,900 \\
\hline $\begin{array}{l}\text { Honey Locust } \\
\text { Gleditsia triacanthos }\end{array}$ & 10.31 & $\begin{array}{l}\text { Industrial or Large } \\
\text { Commercial Business }\end{array}$ & 829 & 32 & $829 \times 32=$ & 26,528 \\
\hline $\begin{array}{l}\text { American Sycamore } \\
\text { Platanus occidentalis }\end{array}$ & 3.58 & $\begin{array}{l}\text { Industrial or Large } \\
\text { Commercial Business }\end{array}$ & 124 & 2 & $124 \times 2=$ & 248 \\
\hline $\begin{array}{l}\text { Norway Maple } \\
\text { Acer platanoides }\end{array}$ & 14.64 & $\begin{array}{l}\text { Industrial or Large } \\
\text { Commercial Business }\end{array}$ & 1,354 & 1 & $1,354 \times 1=$ & 1,354 \\
\hline $\begin{array}{l}\text { Kentucky coffeetree } \\
\text { Gymnocladus dioicus }\end{array}$ & 15.6 & $\begin{array}{l}\text { Industrial or Large } \\
\text { Commercial Business }\end{array}$ & 1,578 & 1 & $1,578 \times 1=$ & 1,578 \\
\hline $\begin{array}{l}\text { Swamp White Oak } \\
\text { Quercus bicolor }\end{array}$ & 0.84 & $\begin{array}{l}\text { Industrial or Large } \\
\text { Commercial Business }\end{array}$ & 7 & 4 & $7 \times 4=$ & 28 \\
\hline $\begin{array}{l}\text { American Linden } \\
\text { Tilia americana }\end{array}$ & 17.19 & $\begin{array}{l}\text { Industrial or Large } \\
\text { Commercial Business }\end{array}$ & 1,665 & 1 & $1,665 \times 1=$ & 1,665 \\
\hline $\begin{array}{l}\text { Red Maple } \\
\text { Acer rubrum }\end{array}$ & 11.14 & $\begin{array}{l}\text { Industrial or Large } \\
\text { Commercial Business }\end{array}$ & 968 & 1 & $968 \times 1=$ & 968 \\
\hline \multirow{2}{*}{$\begin{array}{l}\text { Live oak } \\
\text { Quercus } \\
\text { virginiana }\end{array}$} & 3.82 & $\begin{array}{l}\text { Industrial or Large } \\
\text { Commercial Business }\end{array}$ & 137 & 1 & $137 \times 1=$ & 137 \\
\hline & & & & & & $\begin{array}{l}\text { Total Stormwater } \\
\text { Runoff Interception } \\
\text { On Site Per Year } \\
\text { (gal): } 36,576\end{array}$ \\
\hline
\end{tabular}

Table 1.6: Stormwater Runoff Interception Per Year - Existing Street Trees

\begin{tabular}{|l|l|l|l|l|l|l|}
\hline Species & Average DBH (inches) & Land Use & $\begin{array}{l}\text { Stormwater } \\
\text { Runoff } \\
\text { Interception } \\
\text { Per Year (gal) }\end{array}$ & \# of Trees & Calculation & $\begin{array}{l}\text { Total Stormwater } \\
\text { Runoff Interception } \\
\text { by Species Per } \\
\text { Year (gal) }\end{array}$ \\
\hline $\begin{array}{l}\text { Chinquapin Oak } \\
\text { Quercus muehlenbergii }\end{array}$ & 1.40 & $\begin{array}{l}\text { Industrial or Large } \\
\text { Commercial Business }\end{array}$ & 17 & 1 & $17 \times 1=$ & 17 \\
\hline
\end{tabular}




\begin{tabular}{|l|l|l|l|l|l|l|}
\hline $\begin{array}{l}\text { Honey Locust } \\
\text { Gleditsia triacanthos }\end{array}$ & 10.31 & $\begin{array}{l}\text { Industrial or Large } \\
\text { Commercial Business }\end{array}$ & 829 & 19 & $829 \times 19=$ & 15,751 \\
\hline $\begin{array}{l}\text { Norway Maple } \\
\text { Acer platanoides }\end{array}$ & 14.64 & $\begin{array}{l}\text { Industrial or Large } \\
\text { Commercial Business }\end{array}$ & 1,354 & 1 & $1,354 \times 1=$ & 1,354 \\
\hline $\begin{array}{l}\text { American Linden } \\
\text { Tilia americana }\end{array}$ & 17.19 & $\begin{array}{l}\text { Industrial or Large } \\
\text { Commercial Business }\end{array}$ & 1,665 & 1 & $1,665 \times 1=$ & 1,665 \\
\hline $\begin{array}{l}\text { Red Maple } \\
\text { Acer rubrum }\end{array}$ & 11.14 & $\begin{array}{l}\text { Industrial or Large } \\
\text { Commercial Business }\end{array}$ & 968 & 1 & $968 \times 1=$ & 968 \\
\hline & & & & & & $\begin{array}{l}\text { Existing Tree } \\
\text { Rutal Stormwater } \\
\text { On Site Per Year } \\
\text { (gal): 19,755 }\end{array}$ \\
\hline
\end{tabular}

Table 1.7: New Street Tree Stormwater Runoff Interception Per Year

\begin{tabular}{|l|l|l|l|}
\hline $\begin{array}{l}\text { Total Stormwater Runoff Interception } \\
\text { On Site Per Year (gal): }\end{array}$ & $\begin{array}{l}\text { Existing Tree Stormwater Runoff } \\
\text { Interception On Site Per Year (gal) }\end{array}$ & Calculation & $\begin{array}{l}\text { New Tree Stormwater Runoff } \\
\text { Interception On Site Per Year (gal) }\end{array}$ \\
\hline 36,576 & 19,755 & $36,576-19,755=$ & $\mathbf{1 6 , 8 2 1}$ \\
\hline
\end{tabular}

Equivalency for a single-passenger vehicle was calculated using the EPA's Greenhouse Gas Equivalencies Calculator.

Sources:

EPA. 2018. "Greenhouse Gas Equivalencies Calculator," United States Environmental

Protection Agency.

https://www.epa.gov/energy/greenhouse-gas-equivalencies-calculator

Design Workshop. 2016. "Tree Plan."

i-Tree Streets. 2018. "i-Tree Streets." i-Tree Software Suite.

https://www.itreetools.org/streets/

\section{Limitations:}

1. What was inventoried on site had some variation when compared with the tree plan provided by the design firm. Additionally, this calculation does not include carbon sequestration from other plant material on-site.

2. Average species $\mathrm{DBH}$ rather than each individual tree $\mathrm{DBH}$ was used for the calculations. Therefore, the result is an approximation, not an exact value.

- Conserves 2,377 kWh of electricity annually by reducing adjacent buildings' heating and cooling costs through shade and wind protection from existing and newly-planted trees.

Methods:

All tree species on site, both newly planted and original, had their circumference at breast height (4.5 ft. up from the tree base) and location inventoried. Circumference was then translated to average diameter of each species (Table 1.1). To calculate the conserved Kilowatt hours of electricity per year by these trees, i-Tree Planting software was utilized. This tool estimates 
trees' ability to reduce buildings' heating and cooling costs through shading, wind protection, and evapotranspiration. When a specific species was not available to enter, the genus was used instead. The tree species, average $\mathrm{DBH}$, distance to nearest building, side of building, building vintage, climate controls, tree health, and sun exposure were entered into this calculator (see Table 1.8). It should be noted that all building vintage was before 1950, tree health was excellent, and sun exposure was partial. The amount of conserved energy per year was then multiplied by the number of trees on site of that specific species. All totals were added up to obtain a total annual conserved energy value. A calculation of the existing tree count numbers was charted (see Table 1.9) to subtract from the on-site total to find the approximate kilowatt hours conserved by only newly-planted trees (see Table 1.10).

\section{Calculations:}

Table 1.8: Street Tree Kilowatt Hours Conserved and Dollars Saved - Total

\begin{tabular}{|c|c|c|c|c|c|c|}
\hline Species & $\begin{array}{l}\text { Averag } \\
\text { e DBH } \\
\text { (in) }\end{array}$ & $\begin{array}{l}\text { \# of Trees Per Side of } \\
\text { Building }\end{array}$ & $\begin{array}{l}\text { Kilowatt } \\
\text { Hours } \\
\text { Conserved } \\
\text { Per Year } \\
\text { Per } \\
\text { Building } \\
\text { Side }\end{array}$ & $\begin{array}{l}\text { Total Kilowatt } \\
\text { Hours Conserved } \\
\text { Per Species Per } \\
\text { Year }\end{array}$ & $\begin{array}{l}\text { Total Dollars Saved by } \\
\text { Species Per Year (Kilowatt } \\
\text { Hours x } \$ 0.10 \text { ) }\end{array}$ & $\begin{array}{l}\text { Average Kilowatt Hours } \\
\text { Conserved Per Tree Per Year }\end{array}$ \\
\hline $\begin{array}{l}\text { Chinquapin Oak } \\
\text { Quercus } \\
\text { muehlenbergii }\end{array}$ & 1.40 & $\begin{array}{l}N: 1 \\
S: 2 \\
E: 4 \\
W: 3\end{array}$ & $\begin{array}{l}\text { N: } 0.1 \\
\text { S: } 4.4 \\
\text { E: } 20.6 \\
\text { W: } 69.9\end{array}$ & 95 & $\$ 9.50$ & 9.5 \\
\hline $\begin{array}{l}\text { Valley Forge Elm } \\
\text { Ulmus Americana } \\
\text { 'Valley Forge' }\end{array}$ & 4.93 & $\begin{array}{l}\mathrm{N}: 12 \\
\mathrm{~S}: 16 \\
\mathrm{E}: 1 \\
\mathrm{~W}: 1\end{array}$ & $\begin{array}{l}N: 37.3 \\
S: 272.2 \\
E: 20 \\
W: 68.5\end{array}$ & 398 & $\$ 39.80$ & 13.27 \\
\hline $\begin{array}{l}\text { Honey Locust } \\
\text { Gleditsia } \\
\text { triacanthos }\end{array}$ & 10.31 & $\begin{array}{l}N: 12 \\
S: 9 \\
E: 1 \\
W: 10\end{array}$ & $\begin{array}{l}N: 104.1 \\
\text { S: } 362.8 \\
\text { E: } 36.2 \\
\text { W: } 993.6\end{array}$ & $1,496.7$ & $\$ 149.67$ & 46.77 \\
\hline $\begin{array}{l}\text { American } \\
\text { Sycamore } \\
\text { Platanus } \\
\text { occidentalis }\end{array}$ & 3.58 & $\begin{array}{l}S: 1 \\
W: 1\end{array}$ & $\begin{array}{l}\text { S: } 13.8 \\
\text { W: } 64.1\end{array}$ & 77.9 & $\$ 7.79$ & 38.95 \\
\hline $\begin{array}{l}\text { Norway Maple } \\
\text { Acer platanoides }\end{array}$ & 14.64 & $\mathrm{E}: 1$ & E: 65.5 & 65.5 & $\$ 6.55$ & 65.5 \\
\hline $\begin{array}{l}\text { Kentucky } \\
\text { coffeetree } \\
\text { Gymnocladus } \\
\text { dioicus }\end{array}$ & 15.6 & $\mathrm{E}: 1$ & E: 86.3 & 86.3 & $\$ 8.63$ & 86.3 \\
\hline $\begin{array}{l}\text { Swamp White Oak } \\
\text { Quercus bicolor }\end{array}$ & 0.84 & $\begin{array}{l}N: 1 \\
W: 2 \\
E: 1\end{array}$ & $\begin{array}{l}\mathrm{N}: 0.1 \\
\mathrm{~W}: 36.3 \\
\mathrm{E}: 4\end{array}$ & 40.4 & $\$ 4.04$ & 10.1 \\
\hline $\begin{array}{l}\text { American Linden } \\
\text { Tilia americana }\end{array}$ & 17.19 & $\mathrm{E}: 1$ & E: 40.5 & 40.5 & $\$ 4.05$ & 40.5 \\
\hline $\begin{array}{l}\text { Red Maple } \\
\text { Acer rubrum }\end{array}$ & 11.14 & $\mathrm{~N}: 1$ & $\mathrm{~N}: 21.5$ & 21.5 & $\$ 2.15$ & 21.5 \\
\hline \multirow{2}{*}{$\begin{array}{l}\text { Live oak } \\
\text { Quercus } \\
\text { virginiana }\end{array}$} & 3.82 & W:1 & W: 55.4 & 55.4 & $\$ 5.54$ & 55.4 \\
\hline & & & & $\begin{array}{l}\text { Total Kilowatt } \\
\text { Hours Conserved } \\
\text { Per Year: } 2377.2\end{array}$ & $\begin{array}{l}\text { Total Dollars Saved Per Year: } \\
\$ 237.72\end{array}$ & \\
\hline
\end{tabular}


Table 1.9: Kilowatt Hours Conserved and Dollars Saved - Existing Street Trees

\begin{tabular}{|l|l|l|l|l|l|l|}
\hline Species & Average DBH (inches) & $\begin{array}{l}\text { Average } \\
\text { Kilowatt } \\
\text { Hours } \\
\text { Conserved } \\
\text { Per Year }\end{array}$ & $\begin{array}{l}\text { \# of } \\
\text { Trees }\end{array}$ & Calculation & $\begin{array}{l}\text { Total Kilowatt } \\
\text { Hours } \\
\text { Conserved by } \\
\text { Species Per } \\
\text { Year }\end{array}$ & $\begin{array}{l}\text { Total Dollars } \\
\text { Saved by } \\
\text { Species Per } \\
\text { Year (Kilowatt } \\
\text { Hours x \$0.10) }\end{array}$ \\
\hline $\begin{array}{l}\text { Chinquapin Oak } \\
\text { Quercus muehlenbergii }\end{array}$ & 1.40 & 9.5 & 1 & $9.5 \times 1$ & 9.5 & $\$ 0.95$ \\
\hline $\begin{array}{l}\text { Honey Locust } \\
\text { Gleditsia triacanthos }\end{array}$ & 10.31 & 46.77 & 19 & $46.77 \times 19$ & 888.63 & $\$ 88.86$ \\
\hline $\begin{array}{l}\text { Norway Maple } \\
\text { Acer platanoides }\end{array}$ & 14.64 & 65.5 & 1 & $65.5 \times 1$ & 65.5 & $\$ 6.55$ \\
\hline $\begin{array}{l}\text { American Linden } \\
\text { Tilia americana }\end{array}$ & 17.19 & 40.5 & 1 & $40.5 \times 1$ & 40.5 & $\$ 4.05$ \\
\hline $\begin{array}{l}\text { Red Maple } \\
\text { Acer rubrum }\end{array}$ & 11.14 & 21.5 & 1 & $21.5 \times 1$ & 21.5 & $\$ 2.15$ \\
\hline & & & & & $\begin{array}{l}\text { Total Kilowatt } \\
\text { Cours } \\
\text { Conserved: } \\
\mathbf{1 , 0 2 5 . 6 3}\end{array}$ & $\begin{array}{l}\text { Total Dollars } \\
\text { Saved: } \$ 102.56\end{array}$ \\
\hline
\end{tabular}

Table 1.10: Kilowatt Hours Conserved and Dollars Saved - New Street Trees

\begin{tabular}{|l|l|l|l|}
\hline $\begin{array}{l}\text { Total Kilowatt Hours Conserved and } \\
\text { Dollars Saved: }\end{array}$ & $\begin{array}{l}\text { Existing Tree Kilowatt Hours } \\
\text { Conserved and Dollars Saved: }\end{array}$ & Calculation & $\begin{array}{l}\text { New Tree Kilowatt Hours Conserved } \\
\text { and Dollars Saved: }\end{array}$ \\
\hline $\begin{array}{l}\mathbf{2 3 7 7 . 2} \mathbf{~ k W h} \\
\mathbf{2 3 7 . 7 2}\end{array}$ & $\begin{array}{l}\mathbf{1 , 0 2 5 . 6 3} \mathbf{~ k W h} \\
\mathbf{\$ 1 0 2 . 5 6}\end{array}$ & $\begin{array}{l}2,377.2-1,025.63=1,351.57 \\
237.72-102.56=135.16\end{array}$ & $\begin{array}{l}\mathbf{1 , 3 5 1 . 5 7} \mathbf{~ k W h} \\
\mathbf{1 3 3 5 . 1 6}\end{array}$ \\
\hline
\end{tabular}

\section{Sources:}

Design Workshop. 2016. "Tree Plan."

i-Tree Planting. 2018. "i-Tree Planting." i-Tree Software Suite.

https://planting. itreetools.org/app/location/

\section{Limitations:}

1. Average species $\mathrm{DBH}$ rather than each individual tree $\mathrm{DBH}$ was used for the calculations. Therefore, the result is an approximation, not an exact value.

\section{Social Benefits}

- Improves perception of pedestrian safety and comfort for $67 \%$ of 63 surveyed users who had visited the corridor before reconstruction.

\section{Methods:}

By means of a 2018 survey, users who have experienced the P Street Corridor both before and after reconstruction were asked specific comparative questions. One survey question asked 
returning users to indicate whether their perceived level of safety increased or decreased after reconstruction.

Figure 2.2: User Perception of Street Safety. $\quad$ Author: SurveyMonkey

Q16 I feel safer and more comfortable using P Street as a pedestrian after the reconstruction

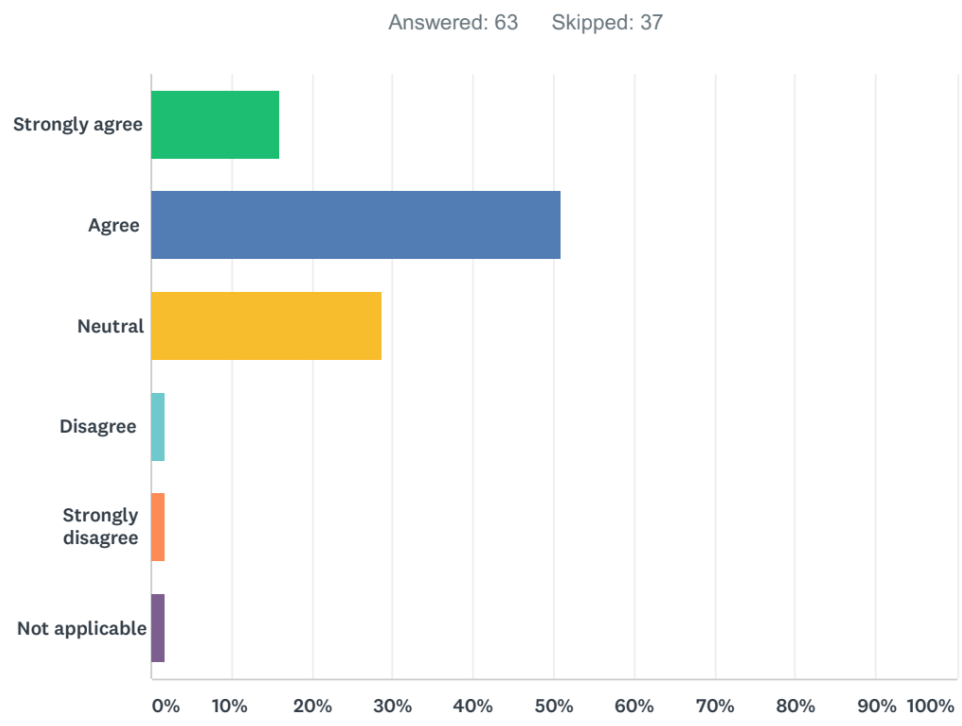

Calculations:

Table 2.2: Returning Users That Feel Safer as Pedestrians

\begin{tabular}{|l|l|l|l|}
\hline "Agree" & "Strongly Agree" & Calculation & "Agree" or "Strongly Agree" \\
\hline $50.79 \%$ & $15.87 \%$ & $50.79+15.87=$ & $66.66 \%$ \\
\hline
\end{tabular}

Sources:

Survey Question 5 in the "Reflecting on the Renovation of P Street" section (see Appendix A).

Limitations:

1. Weather, time of day, and time of year can impact survey responses.

- $\quad$ Reduced street crossing time for pedestrians from an average of 11.5 seconds to 6.9 seconds per crossing, a $40 \%$ reduction.

Methods:

Crosswalk distances and signal times were measured at four locations; P Street (at 12th Street), O Street (at 12th Street), 12th (at P Street), and 12th (at O Street). These measurements are found below (see Table 2.3). At each of these crosswalks, five pedestrians were observed crossing. These crossing times were recorded and averaged (see Table 2.4). Because the pre- 
construction P Street crossing area at 12th Street was approximately the same as O Street is currently, O Street crossing times were measured as a baseline to serve as a proxy for the preproject condition. However, measurements of crossing 12th Street at both P Street and O Street offer a more accurate comparison as it is based upon two conditions on the same roadway. Calculating the percent reduction in crossing times for each comparison yielded the same result: $40 \%$. Finally, crossing distance was divided by average crossing time at 12 th Street on both $P$ and $\mathrm{O}$ in order to identify the average crossing rate at each location.

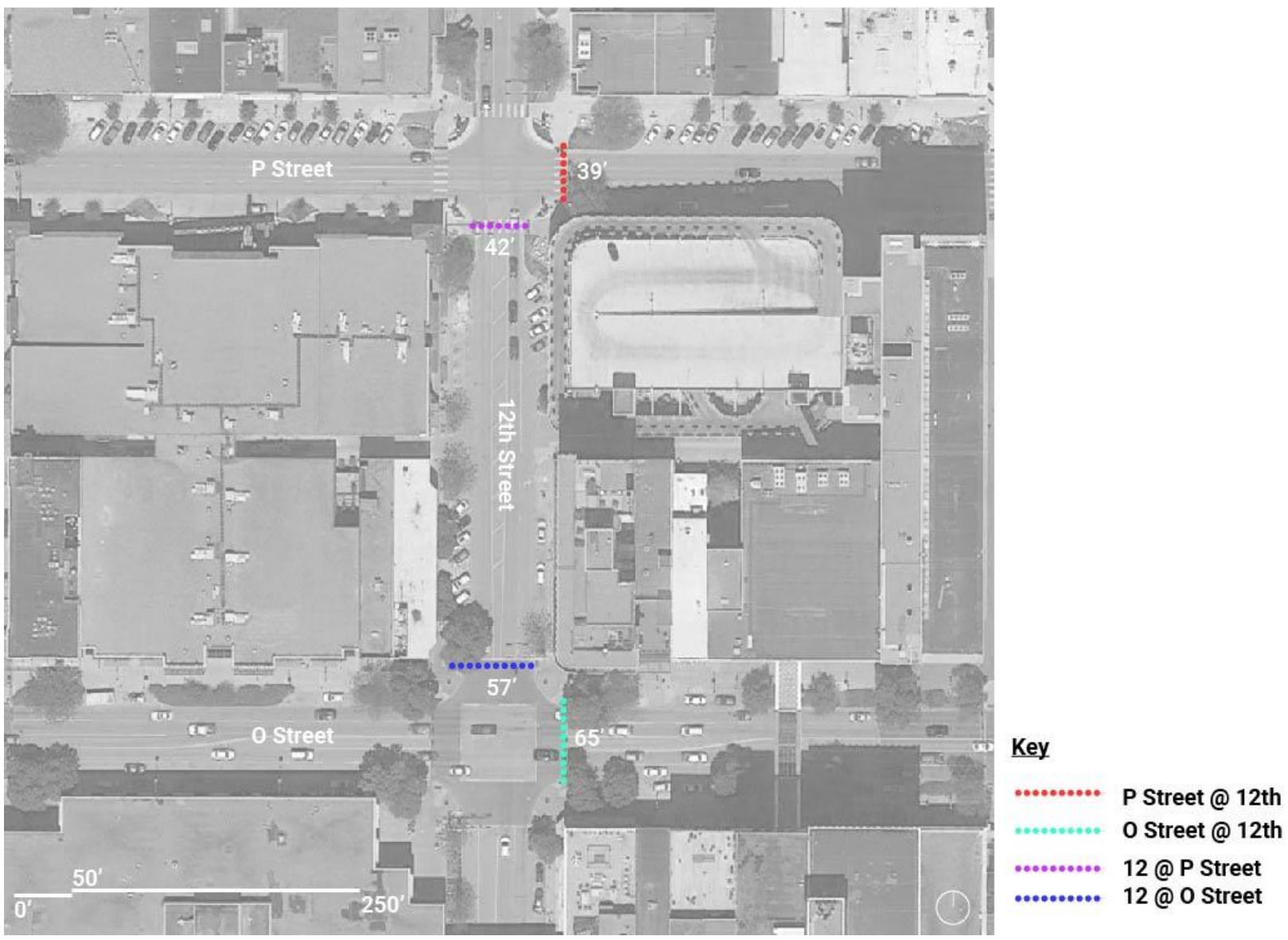

Figure 2.3: Crosswalk Diagram Author: Brandon Zambrano

Calculations:

Table 2.3: Crosswalk Distances

\begin{tabular}{|l|l|l|l|l|}
\hline & P Street @ 12th & O Street @ 12th & 12th @ P Street & 12th @ O Street \\
\hline Distance (ft) & $39^{\prime}$ & $65^{\prime}$ & $42^{\prime}$ & $57^{\prime}$ \\
\hline
\end{tabular}


Table 2.4: Observed User Crossing Times

\begin{tabular}{|l|l|l|l|l|}
\hline & P Street @ 12th & 0 Street @ 12th & 12th @ P Street & 12th @ O Street \\
\hline Trial 1 (sec) & 7 & 14 & 6 & 11 \\
\hline Trial 2 (sec) & 7 & 14 & 8 & 12 \\
\hline Trial 3 (sec) & 8 & 14 & 7 & 11 \\
\hline Trial 4 (sec) & 9 & 11 & 6.5 & 12 \\
\hline Trial 5 (sec) & 8 & 12 & 7 & 11.5 \\
\hline Average (sec) & 7.8 & 13 & 6.9 & 11.5 \\
\hline
\end{tabular}

Table 2.5: Crossing Time Reductions

\begin{tabular}{|l|l|}
\hline Crossing P Street and O Street ${ }^{1}$ & $(13-7.8) / 13 \times 100 \rightarrow \mathbf{4 0} \%$ reduction \\
\hline Crossing 12th @ P Street and O Street ${ }^{2}$ & $6.9 / 11.5=0.6 \rightarrow 1-0.6=0.4 \rightarrow \mathbf{4 0} \%$ reduction \\
\hline
\end{tabular}

1 Previous to construction, $\mathrm{P}$ Street closely resembled $\mathrm{O}$ Street in street width and crosswalks

2 Comparing the crossing times on 12th $\mathrm{St} @$ both $\mathrm{P}$ and $\mathrm{O}$ is a more accurate measurement of crossing time reduction because it compares two crossings on a single roadway

Table 2.6: Rate of Crossing Per Second

\begin{tabular}{|l|l|l|l|}
\hline & Crossing Distance (ft) & Average Crossing Time (sec) & Crossing Rate (ft/sec) \\
\hline Crossing 12th @ O Street & 57 & 11.5 & 4.96 \\
\hline Crossing 12th @ P Street & 42 & 6.9 & 6.09 \\
\hline
\end{tabular}

\section{Sources:}

On-site observations using measuring tape and a digital timer.

\section{Limitations:}

1. Perception of space and safety may change how fast a user walks.

2. Variation in personal habits of pedestrians observed may affect the average walking speed accuracy.

- Improved user perception of the appearance of P Street, with $80 \%$ of 100 surveyed visitors rating the street's appearance as "good" or "very good" as compared to $23 \%$ before the redesign.

\section{Methods:}

The design firm's initial user survey at the time of the Master Plan asked users to rate P Street as either very poor, poor, neutral, good, or very good. By asking the identical question on a 2018 post project survey, a direct comparison of survey results was made. 
Figure 2.1: User Perception Results. $\quad$ Author: SurveyMonkey Q9 How would you rate the appearance of P Street today?

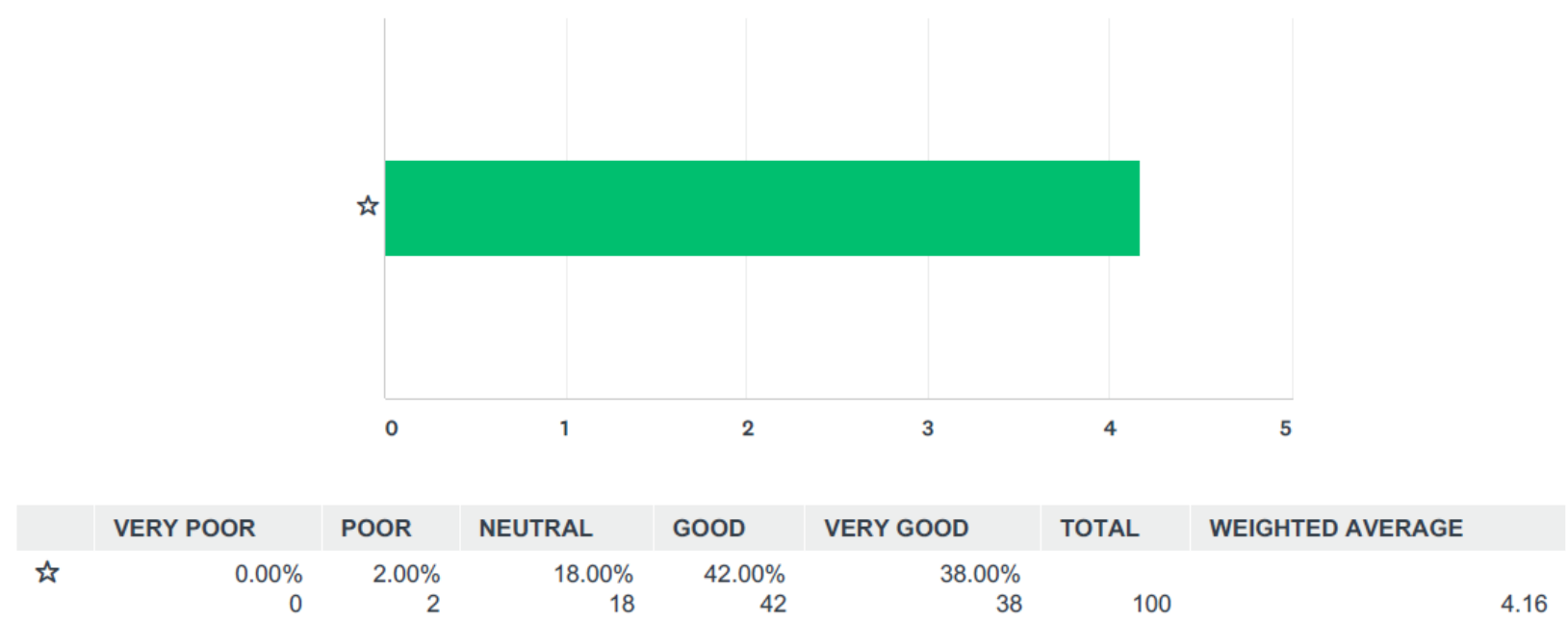

Based on a weighted average, 2018 survey respondents perceived the site $40 \%$ more positively than pre-project survey respondents.

\section{Calculations:}

Table 2.1: Appearance Ratings

\begin{tabular}{|c|c|c|c|c|c|c|c|}
\hline & Very Poor (1) & Poor (2) & Neutral (3) & Good (4) & Very Good (5) & $\begin{array}{l}\text { Weighted Average } \\
\text { Rate Calculation }\end{array}$ & $\begin{array}{l}\text { Weighted } \\
\text { Average Rate } \\
\text { (Scale of } 1 \text { to 5) }\end{array}$ \\
\hline $\begin{array}{l}\text { Pre-project } \\
\text { Survey Results }\end{array}$ & $3 \%$ & $16 \%$ & $57 \%$ & $22 \%$ & $1 \%$ & $\begin{array}{l}(1 \times 3)+(2 \times 16)+(3 \times 57) \\
+(4 \times 22)+(5 \times 1)=299 \\
100=2.99\end{array}$ & 2.99 \\
\hline \multirow[t]{3}{*}{$\begin{array}{l}2018 \text { Survey } \\
\text { Results }\end{array}$} & $0 \%$ & $2 \%$ & $18 \%$ & $42 \%$ & $38 \%$ & $\begin{array}{l}(1 \times 0)+(2 \times 2)+(3 \times 18)+ \\
(4 \times 42)+(5 \times 38)=416 / \\
100=4.16\end{array}$ & 4.16 \\
\hline & & & & & & & $\begin{array}{l}\text { Calculation: } 4.16 \\
-2.99=1.17 \rightarrow \\
1.17 / 2.99= \\
.3913\end{array}$ \\
\hline & & & & & & & $\begin{array}{l}\text { Improvement in } \\
\text { User Perception } \\
\text { Rating: } 39.13 \%\end{array}$ \\
\hline
\end{tabular}

\section{Sources:}

Survey Question 2 in the "Current Use and Perception of P Street" Segment (see Appendix A).

\section{Limitations:}

1. Weather, time of day, and time of year can impact survey responses. 


\section{Economic Benefits}

* Reference Appendix B for a locally-owned street front property methodology with inconclusive results

\section{- $\quad$ Contributed to an $11.2 \%$ increase in assessed value for properties on the P Street Corridor from 2014 to 2018.}

\section{Methods:}

With increased popularity and traffic, property values along the corridor have increased. Obtained from the Lancaster County GIS Viewer, the corridor's private property assessed values were recorded for 2014 (year of construction) and 2018 (see Table 3.2). Properties under construction were not included because of their temporary decrease in property value (this only applied to one property along the corridor). A direct comparison was made between the 2014 and 2018 assessed property values to obtain a percent increase in assessed value (see Table 3.3).

Figure 3.2: Assessed Value Changes for Private Properties

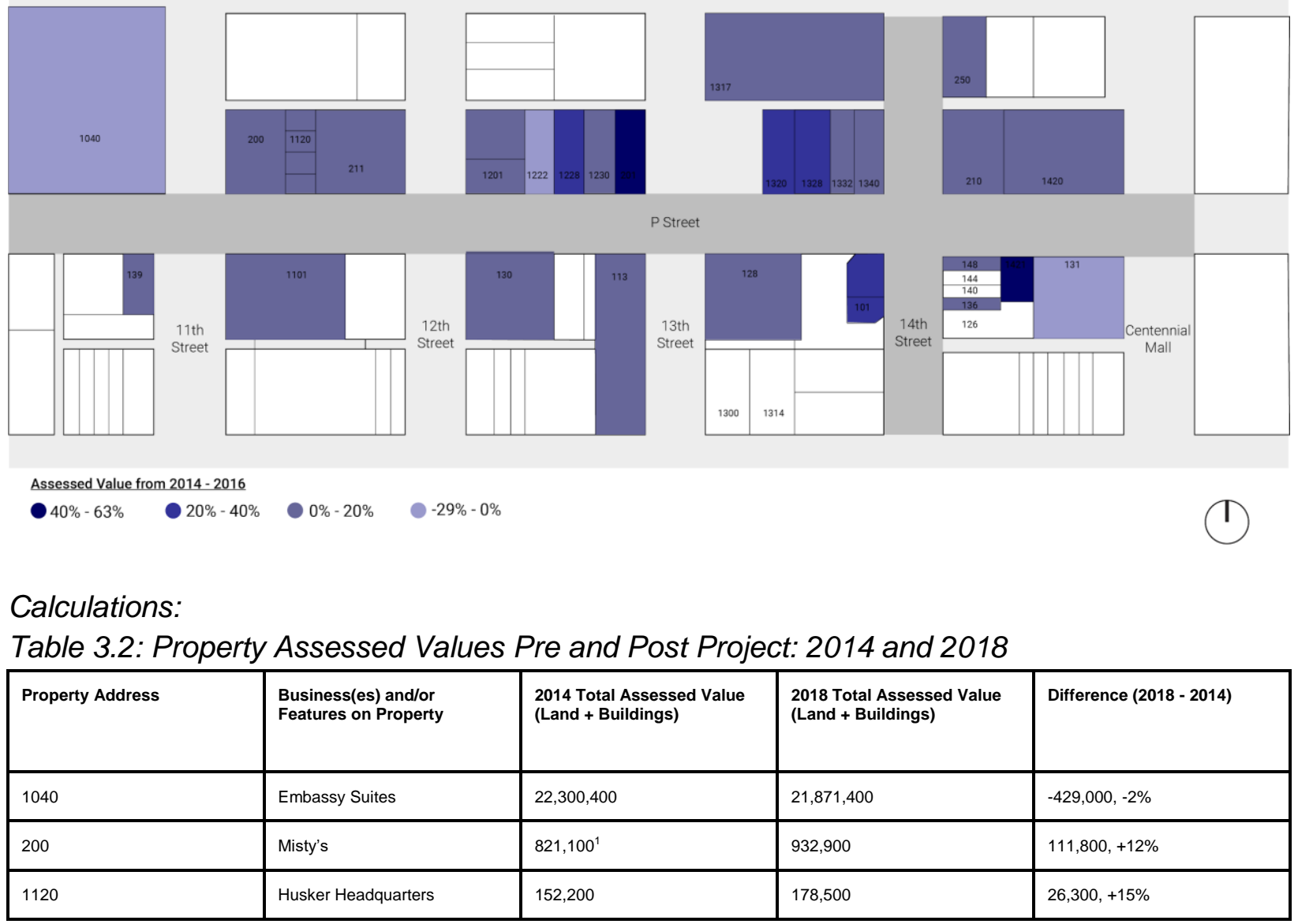




\begin{tabular}{|c|c|c|c|c|}
\hline 211 & $\begin{array}{l}\text { T Mobile } \\
\text { Qdoba } \\
\text { Panera }\end{array}$ & 688,600 & 745,400 & $56,800,+8 \%$ \\
\hline 139 & YMCA & $646,600^{2}$ & 767,900 & $121,300,+16 \%$ \\
\hline 1101 & Marcus Theaters & $7,193,000$ & $7,563,800$ & $370,800,+5 \%$ \\
\hline 1202 & Swanson + Russell & $1,084,500$ & $1,295,800$ & $211,300,+16 \%$ \\
\hline 1228 & $\begin{array}{l}\text { Lan House } \\
\text { Toppers }\end{array}$ & $1,275,100$ & $1,869,600$ & $594,500,+32 \%$ \\
\hline 1230 & Five Guys & 694,500 & 785,000 & $90,500,+12 \%$ \\
\hline 201 & $\begin{array}{l}\text { Fly Fitness } \\
\text { Brown Immigration Law }\end{array}$ & 688,600 & $1,272,200$ & $583,600,+46 \%$ \\
\hline 130 & $\begin{array}{l}\text { Starbucks } \\
\text { Threads Footloose and Fancy }\end{array}$ & $2,444,600$ & $2,636,400$ & $191,800,+7 \%$ \\
\hline 113 & Wells Fargo + Parking Garage & $14,604,000$ & $17,275,000$ & $2,671,000,+15 \%$ \\
\hline 128 & Rococo Theater & $1,139,700$ & $1,192,400$ & $52,700,+4 \%$ \\
\hline 128 & Captain's Chair & 39,500 & 41,800 & $2,300,+6 \%$ \\
\hline 128 & Ruby's Begonias & 39,500 & 41,800 & $2,300,+6 \%$ \\
\hline 101 & Jake's Cigars & 401,300 & 492,100 & $90,800,+18 \%$ \\
\hline 1300 & Tower Square Plaza & $284,000^{2}$ & 511,200 & $227,200,+44 \%$ \\
\hline 1320 & $\begin{array}{l}\text { Operdorse } \\
\text { Bulu Box } \\
\text { Bison Witches } \\
\text { The Coffee House }\end{array}$ & $1,069,900$ & $1,527,000$ & $457,100,+30 \%$ \\
\hline 1328 & Buffalo Wild Wings & 490,100 & 725,800 & $235,700,+32 \%$ \\
\hline 1332 & Stogies Bar & 339,000 & 404,900 & $65,900,+16 \%$ \\
\hline 1340 & $\begin{array}{l}\text { Canes } \\
\text { Foundry Non Profit }\end{array}$ & $1,702,500$ & $1,883,100$ & $180,600,+10 \%$ \\
\hline 1317 & Larson Building & $1,139,700$ & $1,192,400$ & $52,700,+4 \%$ \\
\hline 250 & $\begin{array}{l}\text { NBC Nebraska Bank of } \\
\text { Commerce }\end{array}$ & 588,200 & 692,100 & $103,900,+15 \%$ \\
\hline 210 & $\begin{array}{l}\text { Jersey Mike's Subs } \\
\text { Freezing Thai Rolled Ice } \\
\text { Cream } \\
\text { Chezhay } \\
\text { Noodles and Co } \\
\text { Wahoo's (Vacant) }\end{array}$ & $1,566,300$ & $1,702,800$ & $136,500,+8 \%$ \\
\hline 126 & Honest Abe's & 830,500 & 984,100 & $153,600,+16 \%$ \\
\hline 136 & The Zoo Bar & 114,100 & 135,200 & $21,100,+16 \%$ \\
\hline 148 & The Post and Nickel & 188,600 & 212,600 & $24,000,+11 \%$ \\
\hline \multirow[t]{2}{*}{1421} & 1421 P Street Apts. & 365,300 & 982,500 & $617,200,+63 \%$ \\
\hline & & $\begin{array}{l}\text { 2014 Total Assessed Value: } \\
\$ 62,891,400\end{array}$ & $\begin{array}{l}\text { 2018 Total Assessed Value: } \\
\$ 69,915,700\end{array}$ & $\begin{array}{l}\text { Total Increase in Assessed } \\
\text { Value: } \$ 7,024,300\end{array}$ \\
\hline
\end{tabular}

12017 Value (earliest recorded assessed value available for the property)

22015 Value (earliest recorded assessed value available for the property)

Table 3.3: Increase in Assessed Value 


\begin{tabular}{|l|l|l|l|l|}
\hline 2014 Total Assessed Value & 2018 Total Assessed Value & $\begin{array}{l}\text { Total Increase in Assessed } \\
\text { Value }\end{array}$ & Calculation & $\begin{array}{l}\text { \% Increase in Assessed } \\
\text { Value }\end{array}$ \\
\hline$\$ 62,891,400$ & $\$ 69,915,700$ & $\$ 7,024,300$ & $\$ 7,024,300 / \$ 62,891,400=$ & $.11169=\mathbf{1 1 . 1 7} \%$ \\
\hline
\end{tabular}

Sources:

City of Lincoln/Lancaster County, NE. GIS Viewer: ESRI.

http://maps.lincoln.ne.gov/default/index.html?viewer=GISViewer

\section{Limitations:}

1. Property values along the corridor may have increased due to other factors, such as university housing demands and inflation.

2. A few properties did not have a recorded assessed value for 2014 . Misty's had 2017 as the earliest assessed value. The YMCA and Tower Square Plaza had an earliest recorded value in 2015. These earliest recorded values were entered in place of a 2014 value for these properties, resulting in slightly skewed data.

- Reduced ground floor vacancy rates for street front properties from $5.5 \%$ to $2.3 \%$.

\section{Methods:}

All ground floor street front properties along the P Street Corridor were identified as either "Occupied" or "Vacant" (see Table 3.1) via an on-site inventory observation and internet investigation, with only 1 vacant property identified. One area under construction (location 10 in the chart below) was not considered vacant because there is an intended use upon completion. The total number of vacant properties was divided by the total number of properties to obtain a vacancy rate (see Table 3.2).

A vacancy rate on $\mathrm{P}$ Street of $5.50 \%$ was provided by the primary design firm's pre-project study during the formation of the Master Plan and used the same criteria, with areas under construction not being considered vacant.

\section{Figure 3.1: Ground Floor Vacancy Author: Brandon Zambrano}

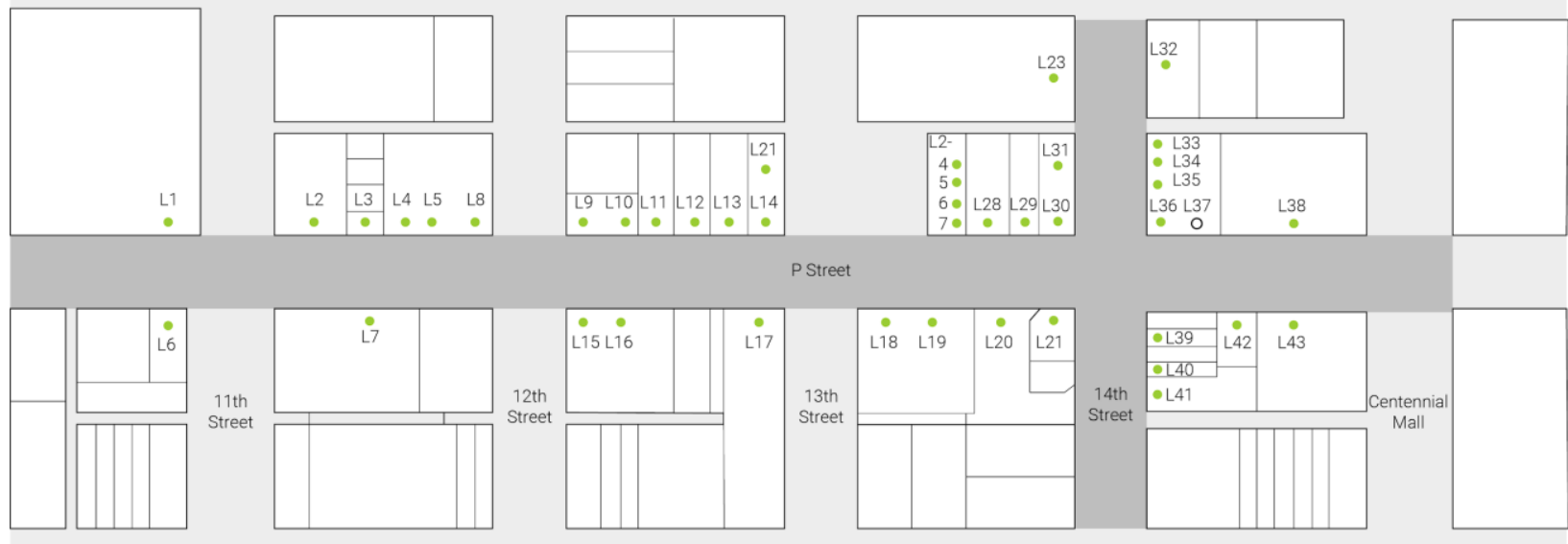

Key

Occupied

$\bigcirc$ Vacant 
Calculations:

Table 3.1: 2018 Vacancy Rate*

\begin{tabular}{|l|l|l|l|}
\hline Total \# of Properties & Total \# of Vacant Properties & Calculation & Vacancy Rate \\
\hline 43 & 1 & $1 / 43=$ & $2.33 \%$ \\
\hline
\end{tabular}

Sources:

Design Workshop. "Pre-Project Study."

Olberding, Matt. 2018. "Bizz Buzz: Restaurant Closings Include Perkins Location, Downtown Taco Shop," Lincoln Journal Star.

https://journalstar.com/blogs/biz-buzz/biz-buzz-restaurant-closings-include-perkins-loc ation-downtown-taco-shop/article 2f831634-e0ce-5105-b28a-a9c6e0b7163c.html

Limitations:

1. The primary design firm's inventoried study area covered more blocks than the final built project, which may impact the overall comparison.

2. Areas under construction were not considered vacant because there is an intended use upon completion. It is unknown how many areas were under construction as part of the primary design firm's evaluation.

\section{Cost Comparison}

- The estimated cost for the materials and installation of a single immature 2" caliper street tree with a modular suspended pavement system is approximately $\$ 5,725$, while traditional installation of the same tree is estimated to cost approximately \$350. However, the appraised value for a mature street tree in good soil conditions as provided by a modular suspended pavement system is approximately 27.4 times higher than at initial planting (from $\$ 350$ initial to $\$ 9,590$ mature) as compared to an increase in value of only 1.5 times for a conventionally planted street tree (from $\$ 350$ initial to $\$ 525$ mature).

Methods:

The added cost of tree planting with a Silva Cell installation was weighed against the increased appraised value of a mature tree planted using Silva Cells vs. the appraised value of a mature tree planted conventionally in smaller tree pits in Lincoln's urban streetscape. In our assessment below, "caliper" is a measurement that describes tree size at planting, and "DBH" (diameter at breast height) is used to document a tree's growth post-installation. 
The local firm on the project, Clark Enersen Partners, provided their cost per cell, average number of cells per street tree, and average cost per new 2" caliper tree in Lincoln, NE (see Table A). To identify the approximate increase in appraised value ${ }^{1}$ for a tree planted in Silva Cells versus a conventionally planted tree, a tree appraisal value chart was created by referencing James Urban's 2008 book Up By Roots. Factors that increased the overall appraised value were determined by using willow oak data in Urban's chart. The willow oak depicted in Urban's chart was selected because of its similarity to P Street's intended canopy size for large shade trees in the form of honey locust and elm along with the site's compacted urban soil conditions (see Table B and Figure A). The factors that affected the increase in appraised value were then combined with Clark Enersen's cost data to calculate approximate appraised values for P Street's street trees with and without the installation of Silva Cells (see Table C).

The installation of a single 2" caliper street tree with a Silva Cell system had an upfront cost in Lincoln in 2014 of approximately $\$ 5,725$. Conventional tree installation (without a Silva Cell system) for a single 2" caliper street tree in Lincoln would have had a much lower upfront cost of approximately $\$ 350$. However, because Silva Cells provide larger soil volumes for street trees that prevent soil compaction, maximize water retention, and increase the lifecycle of an urban tree, the appraised value in mature street trees with Silva Cells is much higher than that of conventionally planted trees without Silva Cells. Mature urban street trees that have grown in better soil conditions have a higher DBH (approximately 3 times larger), larger canopy (approximately double in size), lower rate of replacement, and increased ability to manage stormwater. The appraised value for a mature street tree in good soil conditions, such as that achieved through the use of Silva Cells, is approximately 27.4 times higher than at initial planting $(\$ 9,590)$ as compared to an increase of only 1.5 times in a conventionally planted street tree (\$525). Although the upfront cost with Silva Cell installation was much higher, the City of Lincoln felt that the higher tree canopy, faster growth rates, increased lifecycle, and higher aesthetic value made this Silva Cell installation a worthwhile investment.

1. Appraised value was determined based upon the International Society of Arboriculture Guide to Plant Appraisal. The appraisal process has many variations based on the uniqueness of each case. Therefore, the appraised values referenced in this cost comparison are generalized to convey an approximate appraisal increase. Reference the full appraisal guide below in "Sources."

\section{Calculations:}

Table A: Base Tree and Silva Cell Cost Data ${ }^{1}$

\begin{tabular}{|l|l|}
\hline Average \# of Silva Cells per tree $^{2}$ & 43 \\
\hline Cost per cell & $\$ 125$ \\
\hline $\begin{array}{l}\text { Average cost for tree and installation per new 2" caliper } \\
\text { tree in Lincoln, NE }\end{array}$ & $\$ 350$ \\
\hline
\end{tabular}

1 The data presented in this chart comes from The Clark Enersen Partners, local firm for the P Street project 2 A range of $40-46$ cells was installed per tree with 1,530 cells installed total

Table B: Appraised Value Increase of Willow Oak 3.5" Caliper in a Commercial District ${ }^{1}$

\begin{tabular}{|l|l|l|}
\hline & Appraised Value & Increase in Appraised Value from Newly Planted to Mature \\
\hline Newly planted & $\$ 720$ & $----_{-}$ \\
\hline
\end{tabular}




\begin{tabular}{|l|l|l|}
\hline Mature with poor soil conditions (10" caliper) & $\$ 1,100$ & $1.5 x$ \\
\hline Mature with good soil conditions (30" caliper) & $\$ 19,700$ & $27.4 x$ \\
\hline
\end{tabular}

1 The appraised values of Willow Oak 3.5" caliper were obtained from James Urban's 2008 book Up by Roots. These appraisal increase factors of the Willow Oak most closely represent the urban street conditions of $\mathrm{P}$ Street along with the intended canopy of large shade trees. Below is the chart from Up by Roots

Figure A: Tree Appraisal Value Affected by Soil Conditions ${ }^{1}$

Table 2.8.2. Appraised value of various trees as affected by soil conditions. (Appraisals prepared by Keith Pitchford using the Guide for Plant Appraisal 2005 values.)

\section{Description}

Appraised value

\section{Newly planted tree}

- Willow oak, 3.5-inch dbh, in good condition in a residential front yard

- Sinqle-stemmed crabapple, 3.5-inch dbh, in good condition in a residential front vard

- Willow oak, 3.5-inch dbh, in good condition; street tree in a commercial district

- Single-stemmed crabapple, 3.5-inch dbh, in good condition; street tree in a commercial district

\section{Mature tree at site with good soil conditions}

- Willow oak, 30-inch dbh, in good condition in a residential front yard

- Single-stemmed crabapple, 15-inch dbh in good condition in a residential front yard

- Willow oak, 30-inch dbh, in good condition; street tree in a commercial district

- Single-stemmed crabapple, 15-inch dbh, in good condition; street tree in a commercial district

Mature tree at sites with poor soil conditions

These trees were assumed to grow at a much slower rate and to be in a stressed condition.

- Willow oak, 10-inch dbh, in poor condition in a residential front yard

- Single-stemmed crabapple, 8-inch dbh, in poor condition in a residential front yard

- Willow oak, 10-inch dbh, in poor condition; street tree in a commercial district

- Single-stemmed crabapple, 8-inch dbh, in poor condition; street tree in a commercial district

1 Urban, James. 2008. "Up By Roots," International Society for Arboriculture

Table C: P Street Tree Appraised Value Calculations

\begin{tabular}{|l|l|l|}
\hline Step & Calculation & Outcome \\
\hline $\begin{array}{l}\text { 1. Appraised value increase factor for poor soil conditions x cost of new 2" caliper tree } \\
\text { with installation (without Silva Cells) }\end{array}$ & $1.5 \times \$ 350$ & $=\$ 525$ \\
\hline $\begin{array}{l}\text { 2. Appraised value increase factor for good soil conditions x cost of new 2" caliper tree } \\
\text { (with Silva Cells) }\end{array}$ & $27.4 \times \$ 350$ & $=\$ 9,590$ \\
\hline
\end{tabular}

\section{Sources:}

Council of Tree and Landscape Appraisers. 2017. "Guide to Plant Appraisal 10th Edition (Draft)," International Society of Arboriculture. https://www.isa-arbor.com/Portals/0/Assets/PDF/News/2017-01-18-draft-10th-edition-g uide-for-plant-appraisal.pdf

Pitchford Associates. 2018. "Tree Inventories/Appraisals," Pitchford Associates Arboriculture + Environmental Consulting. http://pitchfordtrees.com/?services=inventories

Urban, James. 2008. "Up By Roots," International Society for Arboriculture.

Urban, James, and Leda Marritz. 2015. "Growth Rates and Performance of Trees in Silva Cells," DeepRoot Green Infrastructure. https://www.deeproot.com/silvapdfs/resources/SC2/articles/Growth-Rates-and-Perform 


\section{ance-of-Trees-in-Silva-Cells.pdf}

Limitations:

The cost comparison above is an approximation, not an exact calculation, for various reasons:

1. Because no willow oaks were planted on P Street, the factors influencing the overall appraised value are not exact. They are instead based upon similar street conditions (commercial district with compacted soils) and intended canopy (comparable canopy size to the trees on P Street; honeylocust and elms).

2. The methodology described in the International Society of Arboriculture Guide to Plant Appraisal is very flexible and unique case by case. Therefore the factors playing into the $\$ 750$ starting appraisal value for a newly planted willow oak may not match the factors contributing to the initial $P$ Street tree value (combination of tree cost and installation cost). Instead, the significance of this cost comparison is based primarily upon the factor increase rather than the starting values.

3. Installation costs of the Silva Cells are not considered in calculations.

\section{Appendix A: P Street Survey and Results}

\section{P STREET [between $11^{\text {th }}$ street and Centennial Mall] USER SURVEY}

By selecting this box, I agree to participate in this survey and am aware that my personal information will not be collected.

\section{ABOUT YOU}

- $\quad$ Select your age group:

○ $18-25$

- $26-35$

○ $36-45$

○ 46-55

○ $56-65$

○ $65+$

- I have lived in the Lincoln area for...

- 3 years or less

- 3 to 5 years

- 5 to 10 years

- 10 to 20 years

- More than 20 years

- I am visiting from out of town

- Select one of the following as your best descriptor:

- I am a student at UNL

- I am a faculty or staff member at UNL

- I work downtown

- I live downtown

- I live outside of the downtown but I shop downtown

- I frequent the museums and entertainment venues downtown

- I'm visiting from out of town

- What time of day do you typically visit P Street? (select all that apply)

- Early Morning: 6am-10am

○ Late Morning: 10am-12pm 
- Early Afternoon: $12 \mathrm{pm}-2 \mathrm{pm}$

- Late Afternoon: 3pm-5pm

- Evening: $5 \mathrm{pm}-7 \mathrm{pm}$

- Night: 8pm-12am

- Other:

- When you visit P Street, how long do you typically stay?

- 15 minutes or less

- Half hour

- 1-2 hours

o $3+$ hours

o Other:

- I mostly travel to P Street via...

- Bike

- Car

- Bus

- Skateboard

- On foot (Walking)

- On foot (Running/Jogging)

- Other:

- Once I get to P Street, I move around...

- On foot

- By bike

- By car

- By skateboard

- Other:

\section{CURRENT USE AND PERCEPTION OF P STREET}

- I utilize P Street and the surrounding district to... (select all that apply)

- Dine

- Visit bar and entertainment venues (nightlife)

- Shop

- Get to the Haymarket

- Visit family oriented venues (museums, theaters, etc)

- Run errands

- Work

- I live here

- I own a business here

- I own property here

- Worship

- Hang out and people watch

- Pass through on my way elsewhere

Specify:

- How would you rate the appearance of P Street today?

- Very good

- Good

- Neutral

- Poor

- Very Poor

- Why did you give $\mathrm{P}$ Street this rating?

- If neutral or less, what would improve your perception of P Street?

\section{REFLECTING ON THE RENOVATION OF P STREET}


- $\quad$ Are you familiar with what P Street looked like before the 2014 renovation?

- Yes

- No

If you answered "Yes," please continue to fill out the following questions. If you answered "No," please skip to the next section titled "VEHICULAR PARKING"

- Do you find walking on P Street easier or more difficult after the reconstruction? How much easier or more difficult? Circle one.

○ (Much More Difficult) $-5 \quad-4-3 \quad-2 \quad-1 \quad 0 \quad 122345$ (Much Easier)

- Not Applicable

- Do you find biking on $\mathrm{P}$ Street easier or more difficult after the reconstruction? How much easier or more difficult? Circle one.

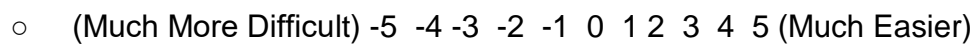

- Not Applicable

- Do you find driving on $\mathrm{P}$ Street easier or more difficult after the reconstruction? How much easier or more difficult? Circle one.

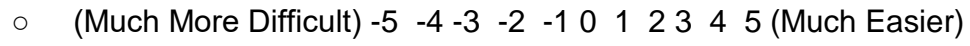

- Not Applicable

- I feel safer and more comfortable using $\mathrm{P}$ Street as a pedestrian after the reconstruction

- Strongly agree

- Agree

- Neutral

- Disagree

- Strongly Disagree

- Other:

- Not Applicable

- I feel safer and more comfortable using P Street as a biker after the reconstruction

- Strongly agree

- Agree

- Neutral

- Disagree

- Strongly Disagree

o Other:

- Not Applicable

- I feel safer and more comfortable using P Street as a driver after the reconstruction

- Strongly agree

- Agree

- Neutral

- Disagree

- Strongly Disagree

o Other:

- Not Applicable

- How often do you shop on P Street after the reconstruction?

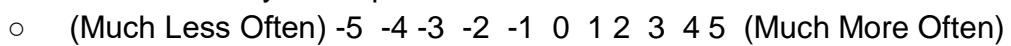

- Not Applicable

- How often do you dine on $\mathrm{P}$ Street after the reconstruction?

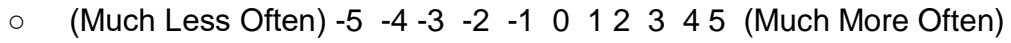

- Not Applicable

- How is the traffic after the reconstruction?

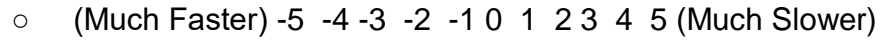

- Not Applicable

- The single most important improvement made to P Street was...

- New plantings (trees, planting beds, etc) 
- New street furniture (benches, bike racks, etc)

- Safer crosswalks

- Better signage and wayfinding

- Better lighting

- More on-street parking

- Dedicated bicycling space in roadway

- Wider sidewalks

- Traffic calming

- Other:

\section{VEHICULAR PARKING}

- Within the P Street District, I feel the quantity of parking is...

- Sufficient along P Street

- Should be reduced along $P$ Street to add more public space for pedestrian activities like outdoor eating/cafes

- Should be increased for commercial purposes along P Street

- Should be increased in the form of parking garages

- I don't know. I would like to learn more.

- Where do you find yourself typically parking?

- Daily parking at a garage

- Monthly parking at a garage

- Best available parking space

- Best available parallel or angled parking space

- Best available angled parking space

- Not applicable as I typically use my bicycle

- Not applicable as I typically walk

- Not applicable as I typically use public transportation

$\circ \quad$ Other

\section{BICYCLING}

If you don't bike on P Street, please skip to the next section titled "PEDESTRIAN ZONE"

- How would you rate your satisfaction with the amount of bike parking on P Street?

- Very good

- Good

- Neutral

- Poor

- Very Poor

- How would you rate your satisfaction with the location of bike parking on P Street?

- Very good

- Good

- Neutral

- Poor

- Very Poor

- If you selected "Poor" or "Very Poor," where would be better locations for the bike parking?

\section{THE PEDESTRIAN ZONE}

- Do you feel safer crossing the street?

- Yes

- No

- I don't know

- When you are walking on P Street, what safety issues concern you most? (select all that apply)

- Crossing 9th and 10th Street

- Not enough points of interest

- Narrow sidewalks 
- Too many driveways

- Fast traffic

- Crossing P Street

- Other

- Crossing other side streets

- Long wait for crossing lights

- Personal security

- How would you rate your satisfaction with the sidewalk widths on P Street? Why?

- Very good

- Good

- Neutral

- Poor

- Very Poor 


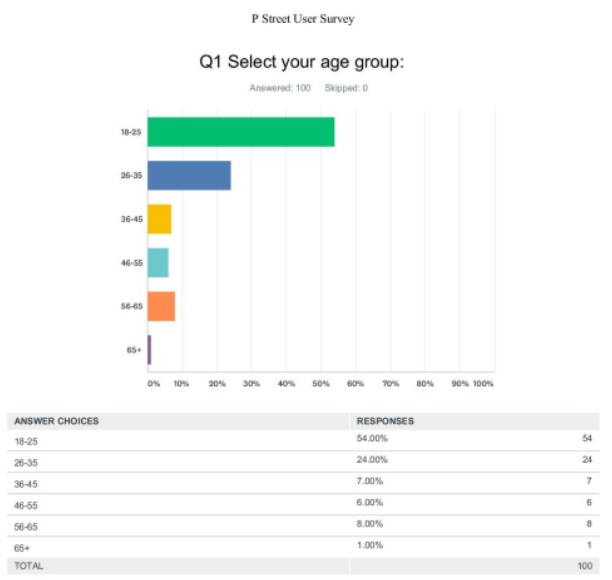

$1 / 32$

Q3 Select one of the following as your best descriptor:

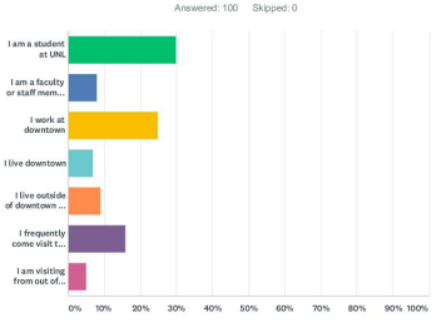

\begin{tabular}{|c|c|}
\hline ANSWER CHOICES & REsPonses \\
\hline Iama student at UNL. & $30.00 \%$ \\
\hline 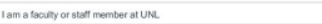 & $8.00 \%$ \\
\hline Inork at townetoon & $25.00 \%$ \\
\hline Ulive comitem & $7.00 \%$ \\
\hline 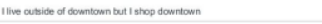 & $8.000 \mathrm{~K}$ \\
\hline 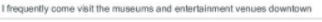 & $16.00 \%$ \\
\hline Iam valing tom ou of of bum & $5.00 \mathrm{~N}$ \\
\hline
\end{tabular}

Q2 I have lived in the Lincoln area for..
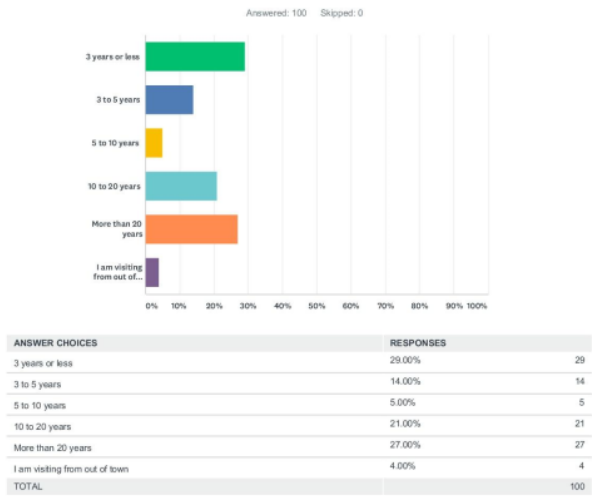

$2 / 32$

Q4 What time of day do you typically visit P street?

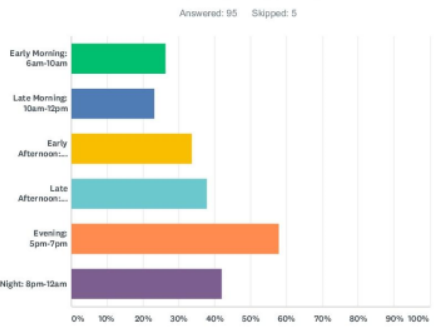

ANSWER CHOCES RESPONSES

Earty Marning tom-10am

Earfy Atomboon: $12 \mathrm{pm}-2 \mathrm{pm}$

Lat Mternoor Sem Som

Evenng Spem-7en

Total Responenersis as

\begin{tabular}{|c|c|}
\hline REsPons & \\
\hline $26.32 \%$ & 25 \\
\hline $23.18 \%$ & 22 \\
\hline $33.68 \%$ & 32 \\
\hline 37.ars & 36 \\
\hline $57.89 \%$ & 55 \\
\hline $42111 \%$ & 40 \\
\hline
\end{tabular}



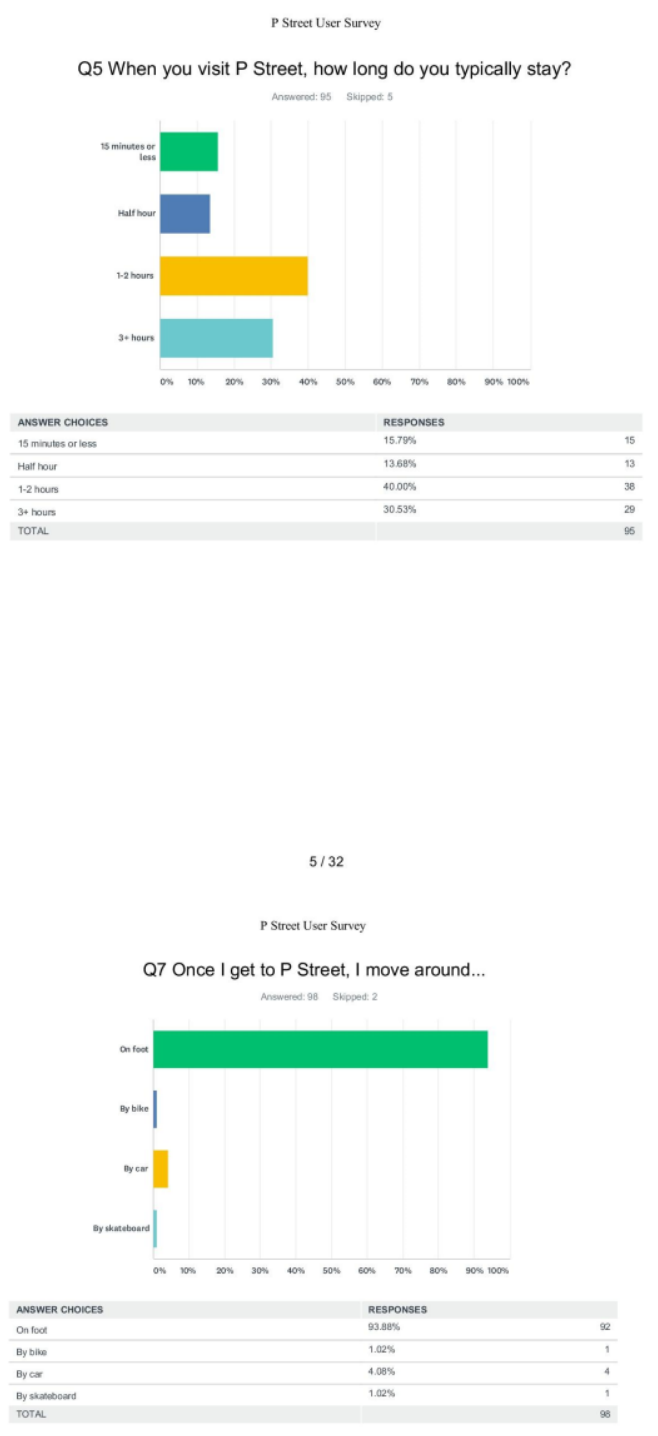

7/32
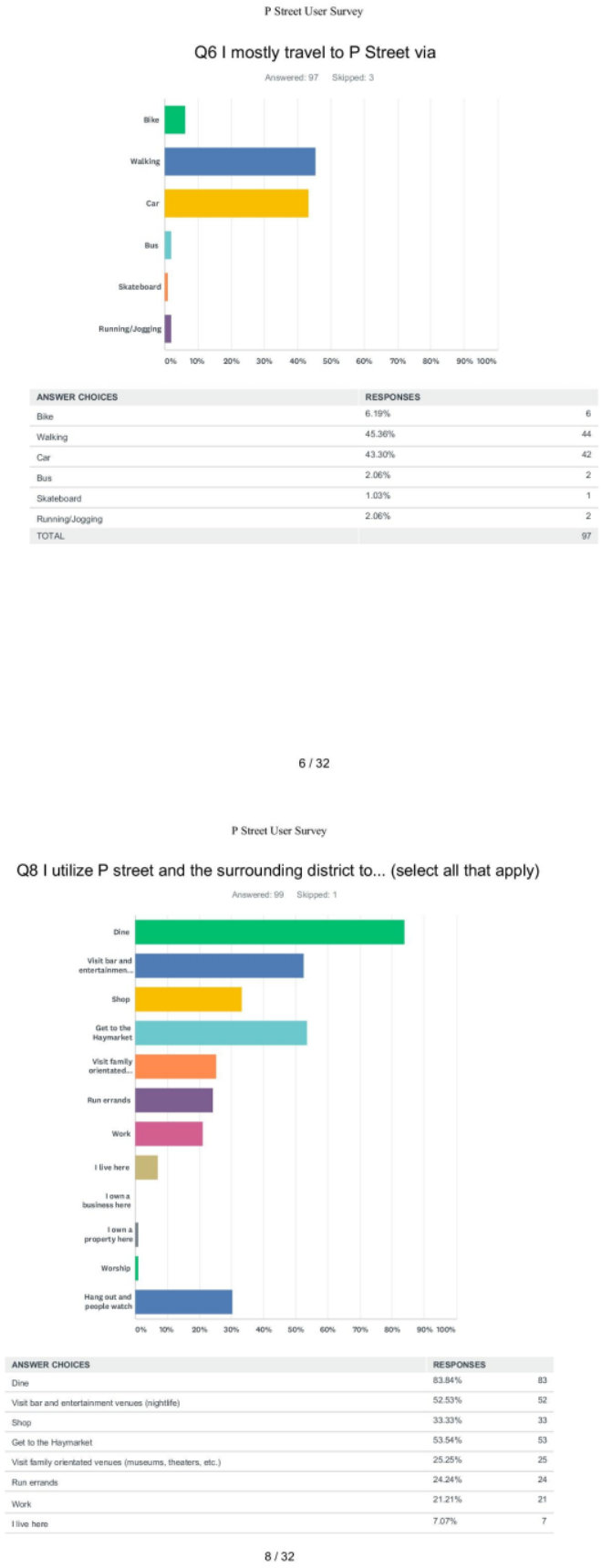
Q9 How would you rate the appearance of P Street today?

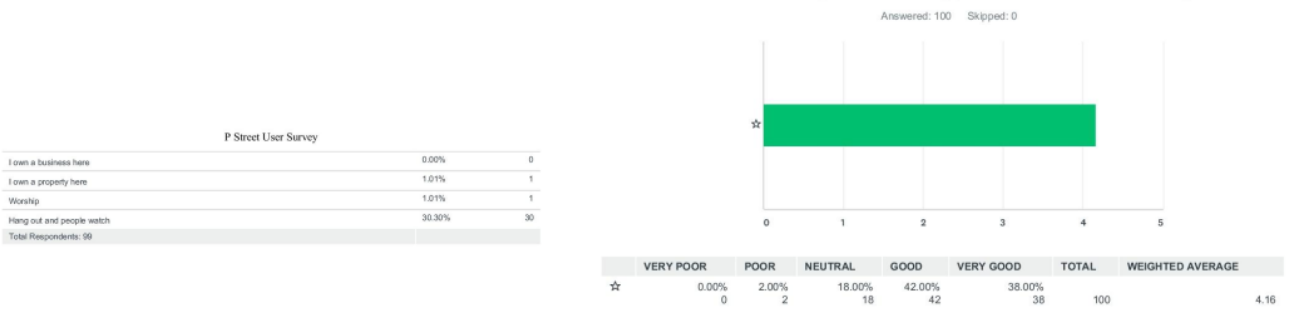

$9 / 32$

P Street User Survey

Q12 Are you familiar with what P Street looked like before the 2014 renovation?

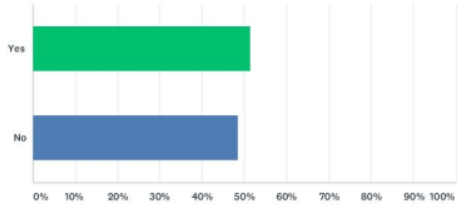

\begin{tabular}{|c|c|c|}
\hline ANSWER CHOICES & RESPONSES & \\
\hline Yes & $51.52 \%$ & 51 \\
\hline No & 48.48\% & 48 \\
\hline
\end{tabular}

$10 / 32$

P Street User Survey

Q13 Do you find walking on $\mathrm{P}$ street easier or more difficult after the reconstruction? -5 being difficult and 5 being easier.

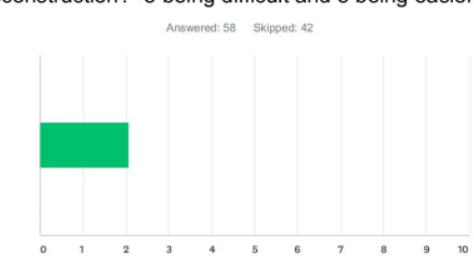

ANSWER CHOICES

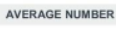

Total Respondents: 58 


\section{P Street User Survey}

Q14 Do you find biking on P street easier or more difficult after the reconstruction? -5 being difficult and 5 being easier.

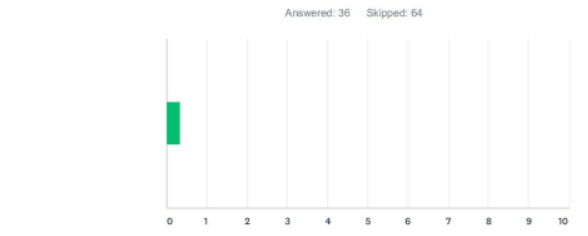

ANSWER CHOICES

AVERAGE NUMBER
$15 / 32$

P Street User Survey

Q16 I feel safer and more comfortable using P Street as a pedestrian after the reconstruction

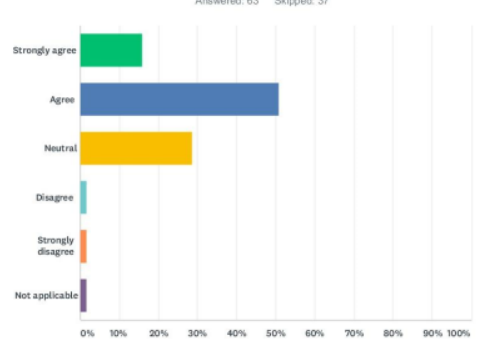

Q15 Do you find driving on $\mathrm{P}$ street easier or more difficult after the reconstruction? -5 being difficult and 5 being easier.

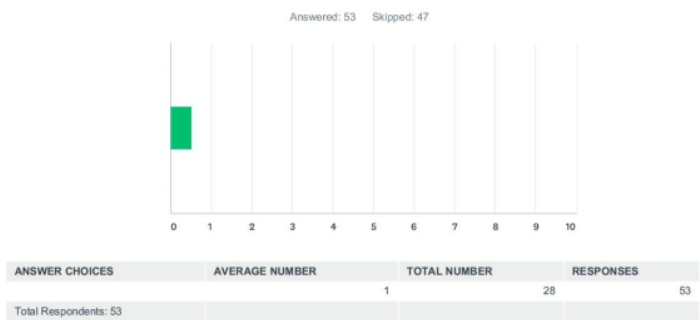

$16 / 32$

P Street User Survey

Q17 I feel safer and more comfortable using P Street as a biker after the reconstruction

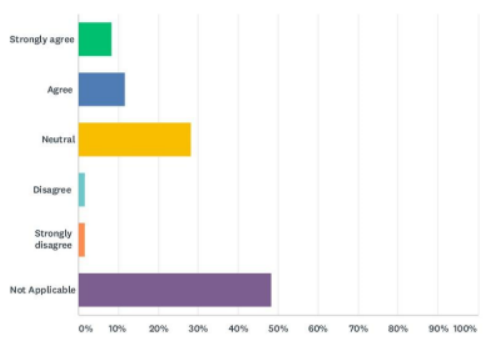

\begin{tabular}{|c|c|}
\hline ANSWER CHOICES & RESPONSES \\
\hline Strongly agree & $8.39 \%$ \\
\hline Apree & $11.67 \%$ \\
\hline Neutral & $28.35 \%$ \\
\hline Disaggree & $1.67 \%$ \\
\hline Strongly disagree & $1.67 \%$ \\
\hline Nat Apolicabile & $48.33 \%$ \\
\hline
\end{tabular}


P Street User Survey

Q18 I feel safer and more comfortable using P Street as a driver after the reconstruction

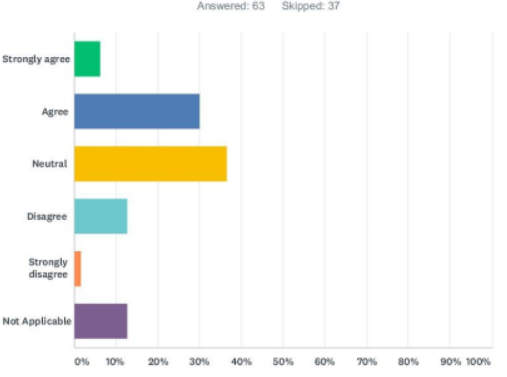

\begin{tabular}{|c|c|c|}
\hline ANSWER CHOICES & RESPONSES & \\
\hline Strongly agree & $6.35 \%$ & \\
\hline Agree & $30.16 \%$ & \\
\hline Neutrad & 36.51\% & 23 \\
\hline Disagree & $1270 \%$ & \\
\hline Strongly disagree & $1.59 \%$ & \\
\hline Net Appicable & $1270 \%$ & \\
\hline
\end{tabular}

P Street User Survey

Q19 How often do you shop on P Street after the reconstruction

ANSWER CHOICES
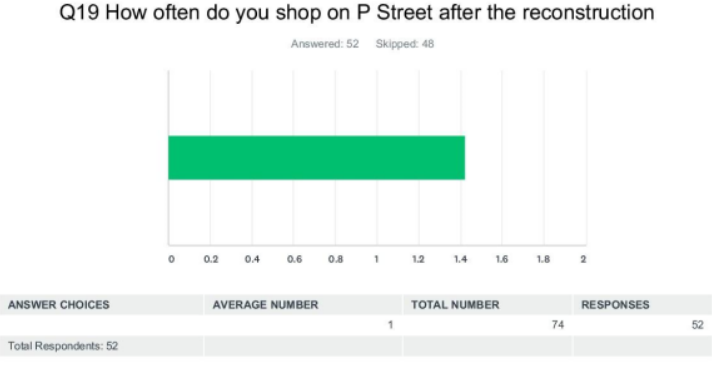

$19 / 32$

P Street User Survey

Q20 How often do you dine on P Street after the reconstruction?

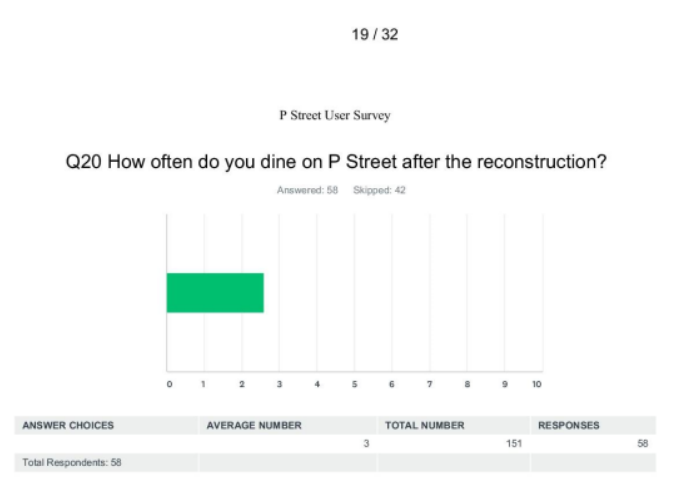

ANSwER CHOICES

AVErage numaer

$21 / 32$

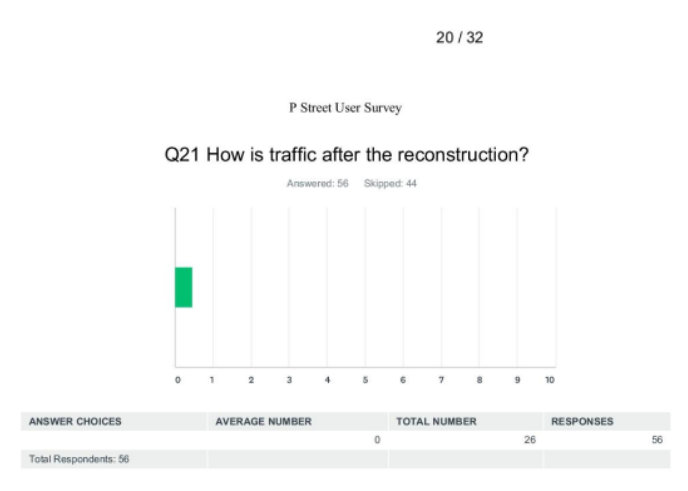


PS Smeat Ueet Sirvey

Q22 The single most important improvement made to P Street was.

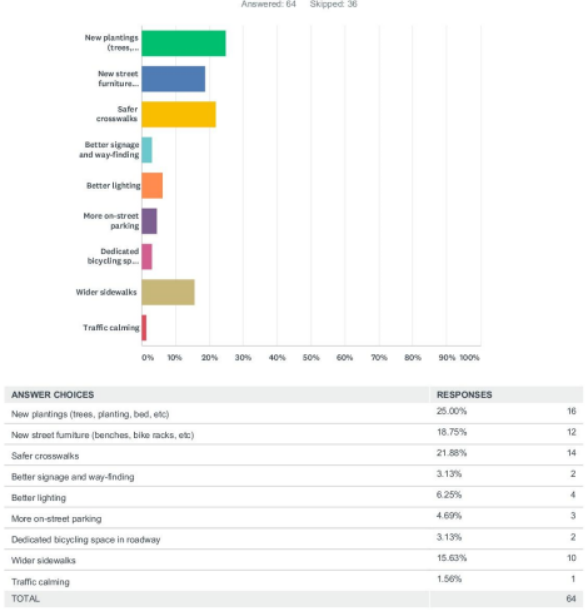

23/32

P Street User Survey

Q24 Where do find yourself typically parking?

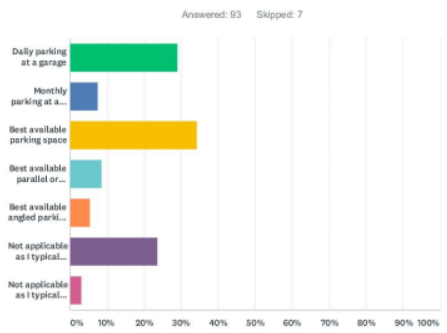

\begin{tabular}{|c|c|c|}
\hline ANSWER CHOICES & RESPONSES & \\
\hline Daly paraxing at a garcopo & $20.03 \%$ & \\
\hline 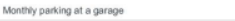 & $7.59 \%$ & \\
\hline Best avalatie paking space & $3.41 \%$ & 32 \\
\hline 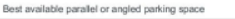 & $8.60 \%$ & \\
\hline 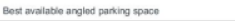 & $538 \%$ & \\
\hline 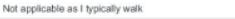 & $23.66 \%$ & ${ }^{22}$ \\
\hline Not applicabilie as i ippicaly use put & $323 \%$ & 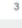 \\
\hline
\end{tabular}

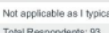

$25 / 32$
Q23 Within the P Street District, I feel the quantity of parking is.
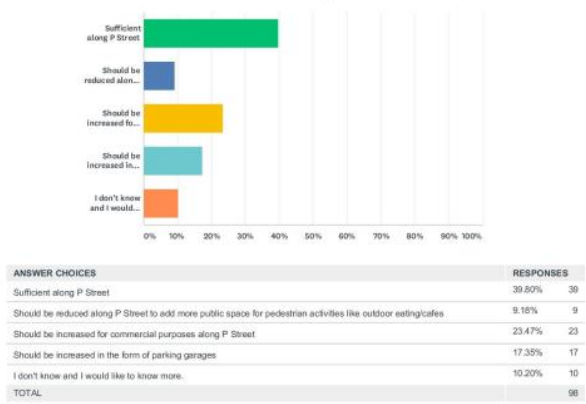

$24 / 32$

P Street User Survey

Q25 How would you rate your satisfaction with the amount of bike parking on P Street?

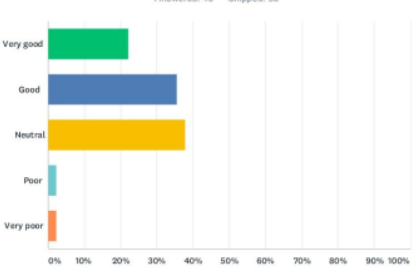

\begin{tabular}{|c|c|c|}
\hline ANSWER CHOICES & RESPONSES & \\
\hline Very pood & 22225 & 10 \\
\hline $\cos$ & $35.56 \%$ & 16 \\
\hline Noutral & 37.785 & 17 \\
\hline Poor & $222 \%$ & 1 \\
\hline very poor & 2228 & 1 \\
\hline
\end{tabular}


P Street User Survey

Q26 How would you rate your satisfaction with the location of the bike parking on $\mathrm{P}$ Street?
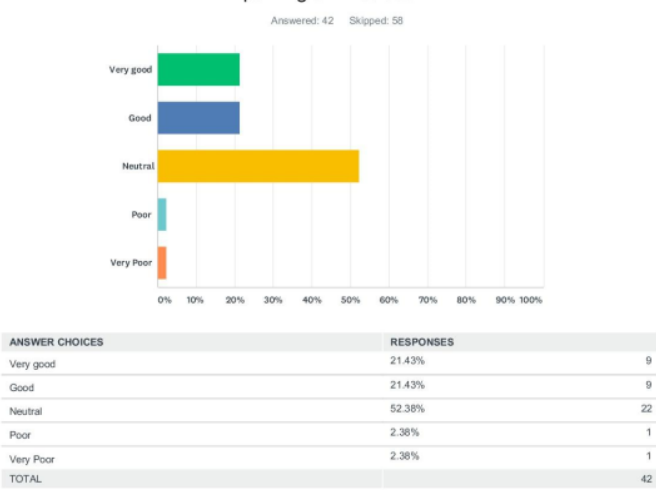

$27 / 32$

P Street User Survey

Q29 When you are walking on P Street, what safety issues concern you most? (select all that apply)

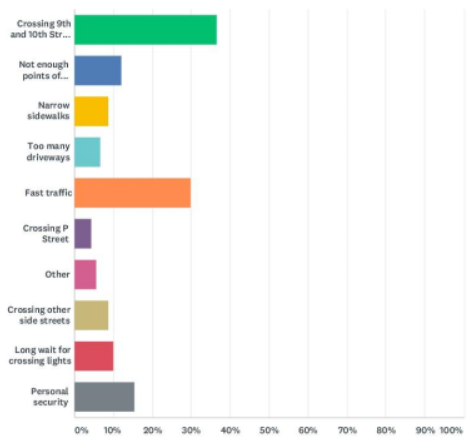

\begin{tabular}{|c|c|c|}
\hline ANSWER CHOICES & RESPONSES & \\
\hline Crossing 9th and 10 Ch Street & $36.67 \%$ & 33 \\
\hline Not enough poins $\alpha$ interest & $1222 \%$ & 11 \\
\hline Narrow sdewalks & $8.89 \%$ & 8 \\
\hline Too many driveways & $6.67 \%$ & 6 \\
\hline Fass traticic & $30.00 \%$ & ${ }^{27}$ \\
\hline Crossing $P$ Street & $4.44 \%$ & 4 \\
\hline other & $5.56 \%$ & 5 \\
\hline Crossing other side stroets & $8.89 \%$ & a \\
\hline Long wat for croosing lights & $10.00 \%$ & 9 \\
\hline
\end{tabular}

$30 / 32$
P Street User Survey

Q28 Do you feel safer crossing the street?
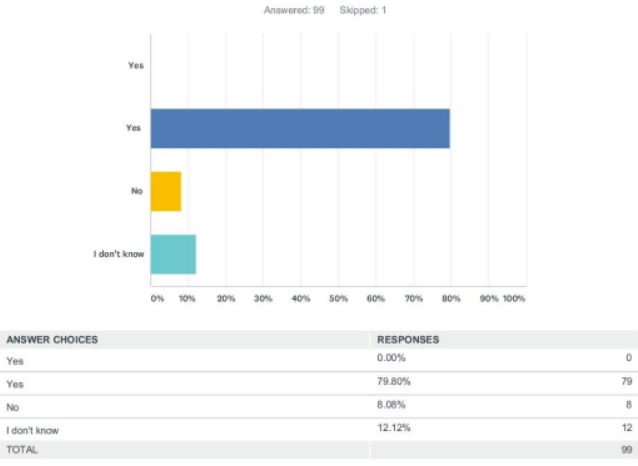

$29 / 32$

P Street User Survey

Q30 How would you rate your satisfaction with the sidewalk widths on P Street? Why?
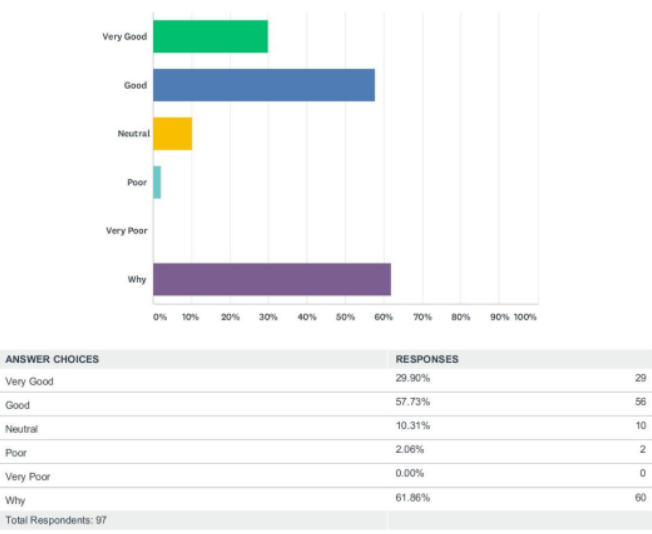

$32 / 32$ 


\section{Appendix B: Locally Owned Street Front, No Significant Benefit Found}

\section{- Increased locally-owned street front presence from $58 \%$ to $58.5 \%$}

\section{Methods:}

P Street Corridor is known for unique locally-owned stores and businesses which give the area a distinct character. Increased pedestrian traffic and dwell time may have lent itself to supporting new local businesses. All businesses along the corridor were recorded as either "Local" or "Chain" (see Table 5.1). Vacant properties and areas under construction were removed from this inventory. A direct comparison was made between a pre and post project count of locally-owned street front businesses (see Table 5.2). The primary design firm recorded a locally owned street front presence of $58 \%$ at the time of the Master Plan.

Figure 5.1: Locally Owned Street Front Presence

Author: Brandon Zambrano

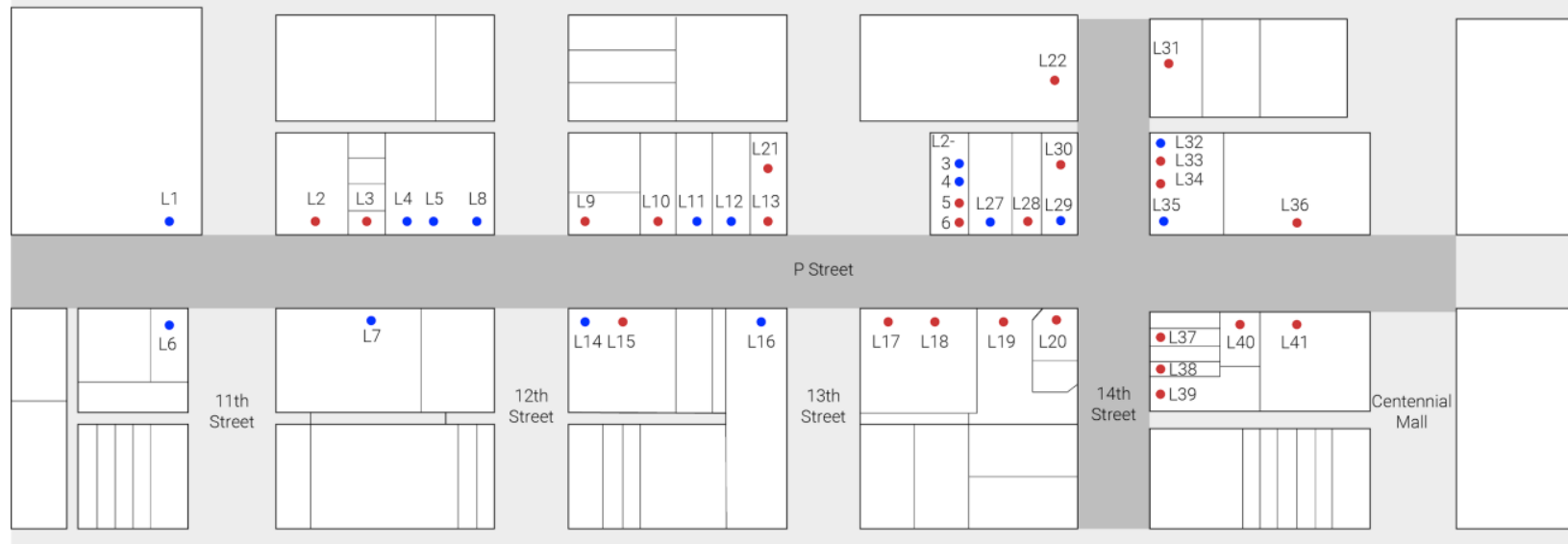

Key

Local

Chain

\section{Calculations:}

Table 5.1: Locally Owned Inventory

\begin{tabular}{|l|l|l|l|}
\hline & Business Name & Local & Chain \\
\hline Location 1 & Embassy Suites & & $x$ \\
\hline Location 2 & Misty's & $x$ & \\
\hline Location 3 & Husker Headquarters & $x$ & \\
\hline Location 4 & T Mobile & & $x$ \\
\hline Location 5 & Qdoba & & $x$ \\
\hline Location 6 & YMCA & & $x$ \\
\hline Location 7 & Marcus Theaters & & $x$ \\
\hline Location 8 & Panera & $x$ \\
\hline
\end{tabular}




\begin{tabular}{|c|c|c|c|}
\hline Location 9 & Swanson + Russell & $x$ & \\
\hline Location 10 & Lan House & $x$ & \\
\hline Location 11 & Toppers Pizza & & $\mathrm{x}$ \\
\hline Location 12 & Five Guys & & $x$ \\
\hline Location 13 & Fly Fitness & $x$ & \\
\hline Location 14 & Starbucks & & $x$ \\
\hline Location 15 & Threads Footloose and Fancy & $x$ & \\
\hline Location 16 & Wells Fargo & & $x$ \\
\hline Location 17 & Rococo Theater & $x$ & \\
\hline Location 18 & Captain's Chair & $\mathrm{x}$ & \\
\hline Location 19 & Ruby's Begonias & $\mathrm{x}$ & \\
\hline Location 20 & Jake's Cigars & $\mathrm{x}$ & \\
\hline Location 21 & Brown Immigration Law & $x$ & \\
\hline Location 22 & Larson Building & $\mathrm{x}$ & \\
\hline Location 23 & Opendorse & & $x$ \\
\hline Location 24 & Bulu Box & & $\mathrm{x}$ \\
\hline Location 25 & Bison Witches & & $x$ \\
\hline Location 26 & The Coffee House & $x$ & \\
\hline Location 27 & Buffalo Wild Wings & & $x$ \\
\hline Location 28 & Stogies Bar & $\mathrm{x}$ & \\
\hline Location 29 & Canes & & $x$ \\
\hline Location 30 & Foundry Non-profit & $x$ & \\
\hline Location 31 & NBC Nebraska Bank of Commerce & $\mathrm{x}$ & \\
\hline Location 32 & Jersey Mike's Subs & & $x$ \\
\hline Location 33 & Freezing Thai Rolled Ice Cream & $\mathrm{x}$ & \\
\hline Location 34 & Chezhay & $x$ & \\
\hline Location 35 & Noodles and Co & & $\mathrm{x}$ \\
\hline Location 36 & Lincoln Children's Museum & $x$ & \\
\hline Location 37 & The Post and Nickel & $x$ & \\
\hline Location 38 & The Zoo Bar & $x$ & \\
\hline Location 39 & Honest Abe's & $x$ & \\
\hline Location 40 & 1421 P Street Apts. & $x$ & \\
\hline \multirow[t]{2}{*}{ Location 41} & Nebraska History Museum & $x$ & \\
\hline & & $\begin{array}{l}\text { Total \# of Locally Owned } \\
\text { Businesses: } 24\end{array}$ & Total \# of Chains:17 \\
\hline
\end{tabular}


Table 5.2: Locally Owned Streetfront Presence

\begin{tabular}{|l|l|l|}
\hline Total \# of Locally Owned Street fronts: & Total \# of Street fronts: & \% of locally owned streetfront \\
\hline 24 & 41 & $58.5 \%$ \\
\hline
\end{tabular}

Sources:

Design Workshop Pre-project Inventory

Limitations:

1. The baseline study area was much larger than the built project.

\section{Appendix C: 2018 Vacancy Status}

Table 6.1: 2018 Vacancy Status

\begin{tabular}{|c|c|c|c|}
\hline & Business Name & Occupied & Vacan \\
\hline Location 1 & Embassy Suites & $\mathrm{x}$ & \\
\hline Location 2 & Misty's & $\mathrm{x}$ & \\
\hline Location 3 & Husker Headquarters & $\mathrm{x}$ & \\
\hline Location 4 & T Mobile & $x$ & \\
\hline Location 5 & Qdoba & $\mathrm{x}$ & \\
\hline Location 6 & YMCA & $x$ & \\
\hline Location 7 & Marcus Theaters & $\mathrm{x}$ & \\
\hline Location 8 & Panera & $\mathrm{x}$ & \\
\hline Location 9 & Swanson + Russell & $\mathrm{x}$ & \\
\hline Location 10 & (Apts. under construction) & $\mathrm{x}$ & \\
\hline Location 11 & Lan House & $x$ & \\
\hline Location 12 & Toppers Pizza & $x$ & \\
\hline Location 13 & Five Guys & $\mathrm{x}$ & \\
\hline Location 14 & Fly Fitness & $\mathrm{x}$ & \\
\hline Location 15 & Starbucks & $x$ & \\
\hline Location 16 & Threads Footloose and Fancy & $x$ & \\
\hline Location 17 & Wells Fargo & $x$ & \\
\hline Location 18 & Rococo Theater & $\mathrm{x}$ & \\
\hline Location 19 & Captain's Chair & $\mathrm{x}$ & \\
\hline Location 20 & Ruby's Begonias & $x$ & \\
\hline Location 21 & Jake's Cigars & $\mathrm{x}$ & \\
\hline Location 22 & Brown Immigration Law & $\mathrm{x}$ & \\
\hline
\end{tabular}




\begin{tabular}{|c|c|c|c|}
\hline Location 23 & Larson Building & $\mathrm{x}$ & \\
\hline Location 24 & Opendorse & $x$ & \\
\hline Location 25 & Bulu Box & $x$ & \\
\hline Location 26 & Bison Witches & $\mathrm{x}$ & \\
\hline Location 27 & The Coffee House & $x$ & \\
\hline Location 28 & Buffalo Wild Wings & $\mathrm{x}$ & \\
\hline Location 29 & Stogies Bar & $x$ & \\
\hline Location 30 & Canes & $x$ & \\
\hline Location 31 & Foundry Non-profit & $x$ & \\
\hline Location 32 & NBC Nebraska Bank of Commerce & $x$ & \\
\hline Location 33 & Jersey Mike's Subs & $x$ & \\
\hline Location 34 & Freezing Thai Rolled Ice Cream & $\mathrm{x}$ & \\
\hline Location 35 & Chezhay & $\mathrm{x}$ & \\
\hline Location 36 & Noodles and Co & $x$ & \\
\hline Location 37 & Wahoo's Fish Tacos & & $x$ \\
\hline Location 38 & Lincoln Children's Museum & $x$ & \\
\hline Location 39 & The Post and Nickel & $x$ & \\
\hline Location 40 & The Zoo Bar & $x$ & \\
\hline Location 41 & Honest Abe's & $x$ & \\
\hline Location 42 & 1421 P Street Apts. & $\mathrm{x}$ & \\
\hline \multirow[t]{2}{*}{ Location 43} & Nebraska History Museum & $x$ & \\
\hline & & & Total \# of Vacant Properties: 1 \\
\hline
\end{tabular}

